\title{
Leptogenesis, fermion masses and mixings in a SUSY SU(5) GUT with $D_{4}$ flavor symmetry
}

\section{Miskaoui and M.A. Loualidi}

LPHE, Modeling and Simulations, Faculty of Science, Mohammed V University in Rabat, 10090 Rabat, Morocco

E-mail: m.miskaoui@gmail.com, mr.medamin@gmail.com

ABSTRACT: We propose a model of fermion masses and mixings based on $\mathrm{SU}(5)$ grand unified theory (GUT) and a $D_{4}$ flavor symmetry. This is a highly predictive $4 \mathrm{D} \mathrm{SU}(5)$ GUT with a flavor symmetry that does not contain a triplet irreducible representation. The Yukawa matrices of quarks and charged leptons are obtained after integrating out heavy messenger fields from renormalizable superpotentials while neutrino masses are originated from the type I seesaw mechanism. The group theoretical factors from 24- and 45-dimensional Higgs fields lead to ratios between the Yukawa couplings in agreement with data, while the dangerous proton decay operators are highly suppressed. By performing a numerical fit, we find that the model captures accurately the mixing angles, the Yukawa couplings and the $C P$ phase of the quark sector at the GUT scale. The neutrino masses are generated at the leading order with the prediction of trimaximal mixing while an additional effective operator is required to account for the baryon asymmetry of the universe (BAU). The model is remarkably predictive because only the normal neutrino mass ordering and the lower octant of the atmospheric angle are allowed while the $C P$ conserving values of the Dirac neutrino phase $\delta_{C P}$ are excluded. Moreover, the predicted values of the effective Majorana mass $m_{\beta \beta}$ can be tested at future neutrinoless double beta decay experiments. An analytical and a numerical study of the BAU via the leptogenesis mechanism is performed. We focused on the regions of parameter space where leptogenesis from the lightest right-handed neutrino is successfully realized. Strong correlations between the parameters of the neutrino sector and the observed BAU are obtained.

Keywords: Beyond Standard Model, Discrete Symmetries, GUT, Neutrino Physics

ARXiv EPRINT: 2106.07332 


\section{Contents}

1 Introduction 1

2 Theoretical setup $\quad 4$

3 Charged fermion sector $\quad 6$

4 Neutrino sector $\quad 10$

5 Numerical analysis and results $\quad 13$

$\begin{array}{lll}5.1 & \text { Numerical fits for charged fermion sector } & 13\end{array}$

$\begin{array}{lll}5.2 & \text { Neutrino phenomenology } & 15\end{array}$

6 Leptogenesis 20

$\begin{array}{lll}7 & \text { Summary and conclusion } & 25\end{array}$

$\begin{array}{ll}\text { A Messenger sector } & 27\end{array}$

B $C P$ asymmetry from the charged lepton mixing and $d=6$ Dirac $\begin{array}{ll}\text { operators } & 28\end{array}$

C Some aspects of the dihedral group $D_{4} \quad 30$

D Vacuum alignment of $D_{4}$ flavon doublets $\quad 31$

\section{Introduction}

During the last two decades, neutrino oscillation experiments have presented vigorous measurements of the neutrino mass-squared differences and their mixing angles ${ }^{1}[1,2]$, conflicting the zero mass prediction of the standard model (SM) of electroweak interactions. Besides the precise measurement of the oscillation parameters, the $C P$ violation and the flavor pattern in the quark and lepton sectors along with the fermion mass hierarchies are not firmly established within the SM. Therefore, theoretical investigations beyond the SM are urgently needed to explain the fermion flavor structure. The $C P$ violation is of particular interest especially after the T2K collaboration excluded some values of $\delta_{C P}$ giving rise to a large improvement of the observed antineutrino oscillation probability at $3 \sigma$ confidence level [6]. Moreover, $C P$ violation is one of the essential ingredients among the three conditions presented by Sakharov to explain the observed BAU through the baryogenesis mechanism [7]. The other two conditions being the baryon number violation and the deviation from thermal equilibrium. The reason to search for this in the lepton sector is due to the fact that the SM predictions for the $C P$ violation - which is encoded in the CKM phase $\delta_{\mathrm{CKM}}$ - is insufficient to generate the observed BAU and thus, new sources

\footnotetext{
${ }^{1}$ The global analysis of all available oscillation data can be found in [3-5].
} 
for $C P$ violation beyond the $\mathrm{SM}$ are required. An interesting approach to successfully produce the observed excess of matter over antimatter in the universe is through the leptogenesis mechanism $^{2}$ [13], which relies on the right-handed ( $\left.\mathrm{RH}\right)$ Majorana neutrinos introduced in the context of type I seesaw mechanism [14-18]. In practical terms, this approach requires lepton number violation which arise naturally in type I seesaw models via the Majorana masses of the $\mathrm{RH}$ neutrinos. Then, a lepton asymmetry is generated by the out of-thermal-equilibrium and $C P$ violating decays of these $\mathrm{RH}$ neutrinos that is eventually converted into a primordial baryon asymmetry by means of the SM sphaleron processes [19]. As a result, the three Sakharov conditions are satisfied in this scenario, which is remarkable considering that leptogenesis connects high energy scales where the BAU takes place and neutrino oscillations that take place at low energy scales.

Grand unified theories are the most attractive high-energy completions of the SM that can bridge the experimentally accessible low energies with extremely high energy phenomenon while providing the unification of electromagnetic, weak and strong interactions [20-24]. When combined with supersymmetry (SUSY) [25], GUTs provide a more powerful explanation to some of the open questions in the SM as well as a solution to some of the problems that are not addressed in the minimal non-SUSY GUTs [26]. The simplest realization of such a combination is provided by the SUSY SU(5) model where the unification of the gauge couplings occurs at a scale of approximately $2 \times 10^{16} \mathrm{GeV}$ [27-31]. As a result of unification, the masses of down quarks and charged leptons are generated from a common renormalizable operator leading to Yukawa couplings of same order of magnitude; $y_{e}=y_{d}, y_{\mu}=y_{s}$, and $y_{\tau}=y_{b}$. It is well-known that these equalities are acceptable for the third generation but fails for the remaining ones because of their conflict with the experimental data. The Georgi Jarlskog (GJ) relations $m_{\mu} / m_{s}=3$ and $m_{e} / m_{d}=1 / 3$ generated from a specific renormalizable operator involving a 45-dimensional Higgs $H_{45}$ presented a first example solution to this issue [32]. In contrast to these relations, considering additional Higgs fields in the 24- or 75-dimensional representations of SU(5) gives rise to nontrivial Clebsch-Gordan (CG) factors with new ratios for the first two generations of Yukawa couplings that are preferred phenomenologically; see for instance refs. [33, 34] for ratios derived from dimension 5 and dimension 6 operators in the context of SUSY SU(5). On the other hand, neutrinos are massless in SUSY SU(5) model which implies that the oscillation phenomenon can not be explained within its minimal realization. The simplest way to address this issue is by introducing $\mathrm{RH}$ singlet fermions to generate neutrino masses via the type I seesaw mechanism, while the mixing angles can be determined by invoking the well-known approach of flavor symmetries. Non-Abelian discrete symmetries ${ }^{3}$ are in particular a powerful tool for explaining the mass hierarchies and the mixing of all fermions [40], especially, those with triplet representations. For example, the discrete symmetry $A_{4}$ is widely used in $\mathrm{SU}(5)$ flavor models to explain the patterns of neutrino masses and their mixing; see for instance refs. [40-48]. On the other hand, the discrete groups with doublet representations like $S_{3}$ and $D_{4}$ are less employed in 4D SU(5) GUTs. In fact, a

\footnotetext{
${ }^{2}$ There are several baryogenesis models using different scenarios to explain the BAU, for a review see refs. [8-12].

${ }^{3}$ For reviews on the use of non-Abelian discrete symmetries, see for instance [35-39].
} 
SUSY SU(5) model based on $D_{4}$ symmetry was considered before in ref. [49]; however, the phenomenological implications of both the lepton and quark sectors were lacking. Here, we will show that $D_{4}$ can provide good results regarding the neutrinos as well as the charged fermions flavor structures by allowing the three generations of matter to be unified into the representations 1 and 2 instead of $3 .^{4}$

In this work, we build a predictive model based on SUSY SU(5) GUT supplemented by a $D_{4}$ flavor symmetry suitable for addressing the above mentioned questions. In particular, we show that our construction leads to results for the pattern of fermion masses and mixings that are consistent with the current experimental data. In fact, this is the first phenomenological analysis of the fermion mass and mixing structures within SUSY SU(5) using the dihedral group $D_{4}$. Besides this discrete group, we have added a U(1) symmetry to engineer the invariance of the superpotentials in the quark and lepton sectors, and also to prevent dangerous operators that mediate rapid proton decay. Apart from the usual SUSY SU(5) superfield spectrum, various superfields are added to the model in order to fulfill different tasks. Namely, many messenger fields denoted as $X_{i}$ and $Y_{i}$ are needed to make the model renormalizable, higher dimensional Higgs fields in the 24 and 45 representations required to obtain realistic Yukawa coupling ratios, gauge singlets superfields - the socalled flavons - needed to break the flavor symmetry and structure the fermions mass matrices, and three right-handed neutrinos $N_{i=1,2,3}^{c}$ responsible for the tiny neutrino masses as well as the BAU through the leptogenesis mechanism. ${ }^{5}$ The introduction of all of the above fields with the requirement to keep the effective superpotentials invariant is highly controlled by the group theoretical structure of the $D_{4} \times \mathrm{U}(1)$ flavor symmetry.

In the charged sector, the messenger fields $X_{i}$ and $Y_{i}$ are coupled to the matter fields, the flavon fields and the 24 and 45 Higgs fields. When $X_{i}$ and $Y_{i}$ are integrated out we obtain the effective operators responsible for the quark and lepton Yukawa couplings, which is then followed by the spontaneous breaking of the flavor and gauge symmetries after the flavons and Higgs fields of the $\mathrm{SU}(5)$ - that is the $5, \overline{5}, 24$, and 45 dimensional Higgs fields denoted respectively as $H_{5}, H_{\overline{5}}, H_{24}$ and $H_{45}$ - acquire nonzero vacuum expectation values (VEVs). On the one hand, the specific VEV alignments of the flavons break the $D_{4} \times \mathrm{U}(1)$ symmetry and help shape the fermions mass matrices, leading eventually to the appropriate flavor structure of the quarks and leptons. On the other hand, the CG factors obtained from the VEV structures of $H_{24}$ and $H_{45}$ lead to the following double ratio of the Yukawa coupling of the first and second generation $\frac{y_{\mu}}{y_{s}} \frac{y_{d}}{y_{e}} \simeq 10.12$ which is consistent with experimental data [62].

In the chargeless sector, the neutrino masses are generated at the renormalizable level through the type I seesaw mechanism. The obtained neutrino mass matrix $m_{\nu}$ is described by only three parameters leading to strong constraints among the physical parameters. Moreover, $m_{\nu}$ is invariant under a particular remnant $Z_{2}$ symmetry which is commonly referred to as a magic symmetry [63], indicating that $m_{\nu}$ is diagonalized by the well-

\footnotetext{
${ }^{4}$ As shown in ref. [50], one of the interesting properties of models with the $D_{4}$ group is that it predicts the well-known $\mu-\tau$ symmetry in a natural manner [51-55] by using minimal set of flavon fields.

${ }^{5}$ For leptogenesis models based on type I seesaw mechanism in the framework of SU(5) GUT see, for instance, refs. [57-61] and references therein.
} 
known trimaximal mixing $\left(T M_{2}\right)$ matrix which is consistent with the observed neutrino mixing angles [64-70]. However, the leptogenesis mechanism can not be induced at the renormalizable level given that the neutrino Yukawa coupling matrix is proportional to the identity matrix which leads to a vanishing lepton asymmetry. Therefore, we show that by introducing one effective operator as a correction to the neutrino Yukawa coupling matrix, our model can accommodate successfully the observed BAU via leptogenesis. ${ }^{6}$ Our main results in the neutrino sector are:

- only the normal hierarchy $(\mathrm{NH})$ for neutrino mass spectrum is allowed,

- only the lower octant of the atmospheric angle is allowed,

- the $C P$ conserving values of the Dirac $C P$ phase $\delta_{C P}$ are excluded,

- the predicted values of the effective Majorana mass in neutrinoless double beta decay $(0 \nu \beta \beta)$ are testable at future $0 \nu \beta \beta$ searches, and

- the correlation between the BAU parameter denoted as $Y_{B}$ and the neutrino sector parameters satisfies the experimental bound of the baryon asymmetry from the Planck collaboration [73].

The rest of the paper is organized as follows. In section 2, we present the particle content of the model as well as their transformation properties under the $\mathrm{SU}(5) \times D_{4} \times \mathrm{U}(1)$ symmetry. In section 3, we derive the mass matrices of the charged fermions and give brief comments on the fast proton decay operators within our construction. In section 4, we study the neutrino sector where the analytical expressions of the neutrino masses and mixing parameters are obtained as a function of the model parameters. In section 5, we show that a perfect fit to the fermion masses and mixings can be obtained for all observables. In section 6 , we carry out an analytical and a numerical study of the BAU via the leptogenesis mechanism. A conclusion is given in section 6. Appendix A describes the messenger sector of the model. Appendix B shows that the contribution of the charged leptons and the higher dimensional Dirac operators to the lepton asymmetry is highly suppressed to account for the BAU. Appendix C provides some tools on $D_{4}$ discrete group. Appendix D describes the realization of the vacuum alignment of $D_{4}$ flavon doublets.

\section{Theoretical setup}

In this section, we describe the different sectors of our $\mathrm{SU}(5) \times D_{4} \times \mathrm{U}(1)$ GUT proposal and fix some notations. The chiral sector of the minimal supersymmetric SU(5) model involves matter and Higgs superfields which are both supplemented by extra superfields in

\footnotetext{
${ }^{6}$ This is a known requirement in models predicting the tribimaximal mixing (TBM) at the leading order, see for instance refs. [71, 72].
} 
the present setup. Apart from the usual SUSY SU(5) superfield spectrum, the building blocks of the present model can be classified into four sets:

- (a) a renormalizable messenger sector with messenger fields $X_{i}$ associated to down quarks, charged leptons and neutrinos, and $Y_{i}$ associated to the up quarks; details on this sector is provided in appendix A,

- (b) two additional higher dimensional Higgs fields in the 24 and 45 GUT representations required for gauge symmetry breaking and for generating Yukawa coupling ratios compatible with the data,

- (c) several flavon superfields carrying quantum numbers under the flavor symmetry $D_{4} \times \mathrm{U}(1)$ needed to break the flavor symmetry and structure the fermions mass matrices, and

- (d) three right-handed neutrinos $N_{i=1,2,3}^{c}$ responsible for generating the tiny neutrino masses via the type I seesaw mechanism as well as the BAU through the leptogenesis mechanism.

Recall that the usual matter superfields denoted as $T_{i}=\left(u_{i}^{c}, e_{i}^{c}, Q_{i L}\right)$ and $F_{i}=\left(d_{i}^{c}, L_{i}\right)$ - with $i=1,2,3$ refers to the three generations of matter - fit into the $10_{i}$ and $\overline{5}_{i}$ representations respectively. Recall also that the low energy Higgs doublets $H_{u}$ and $H_{d}$ of the minimal supersymmetric standard model (MSSM) arise from $H_{5}$ and a mixture of $H_{\overline{5}}$ and $H_{\overline{45}}$ respectively. The 45-dimensional Higgs is usually used to produce the GJ relations differentiating between the (2-2) entry of the down quark and charged lepton mass matrices; however, it has been shown in $[33,34]$ that there are many other options which are preferred compared to GJ relations. These alternatives arise from higher-dimensional operators involving essentially higher dimensional Higgs representations. In our proposal, we use $H_{\overline{45}}$ and $H_{24}$ to produce the following ratios of the diagonal Yukawa couplings $y_{e} / y_{d}=4 / 9$ and $y_{\mu} / y_{s}=9 / 2$ which are in perfect agreement with experimental data [62]. The different steps leading to these ratios is elaborated in the next section. The 45dimensional Higgs $H_{\overline{45}}$ satisfy the following relations

$$
\begin{aligned}
& \left(H_{\overline{45}}\right)_{c}^{a b}=-\left(H_{\overline{45}}\right)_{c}^{b a}, \quad\left(H_{\overline{45}}\right)_{a}^{a b}=0 \\
& \left\langle\left(H_{\overline{45}}\right)_{i}^{i 5}\right\rangle=v_{45}, \quad\left\langle\left(H_{\overline{45}}\right)_{4}^{45}\right\rangle=-3 v_{45} \text { with } i=1,2,3
\end{aligned}
$$

where $v_{45}$ is the VEV of $H_{\overline{45}}$. As for the adjoint Higgs $H_{24}$ which is also responsible for breaking the SU(5) group, it develops its VEV along the direction

$$
\left\langle\left(H_{24}\right)_{b}^{a}\right\rangle=\operatorname{diag}\left(1,1,1, \frac{-3}{2}, \frac{-3}{2}\right) v_{24}
$$

All the above superfields carry as well quantum numbers under the $D_{4} \times \mathrm{U}(1)$ group as depicted in table 1 . In this table, $F_{2,3}$ and $N_{3,2}^{c}$ notations stand for $D_{4}$ doublet assignments $\left(F_{2}, F_{3}\right)^{T}$ and $\left(N_{3}, N_{2}\right)^{T}$ respectively. On the other hand, the $D_{4} \times \mathrm{U}(1)$ invariance requires the introduction of several flavon fields in all the sectors of the model. In the up-quark sector, only the top quark mass arises from a tree level Yukawa coupling, the up and 


\begin{tabular}{l||l|l|l|l|l|l|l|l|l|l|l}
\hline & $T_{1}$ & $T_{2}$ & $T_{3}$ & $F_{1}$ & $F_{2,3}$ & $N_{1}^{c}$ & $N_{3,2}^{c}$ & $H_{5}$ & $H_{\overline{5}}$ & $H_{\overline{45}}$ & $H_{24}$ \\
\hline $\mathrm{SU}(5)$ & $10_{1}$ & $1_{2}$ & $10_{3}$ & $\overline{5}_{1}$ & $\overline{5}_{2,3}$ & $1_{1}^{\nu}$ & $1_{3,2}^{\nu}$ & $5_{H_{u}}$ & $\overline{5}_{H_{d}}$ & $\overline{45}_{H}$ & $24_{H}$ \\
\hline$D_{4}$ & $1_{+,-}$ & $1_{+,-}$ & $1_{+,+}$ & $1_{+,+}$ & $2_{0,0}$ & $1_{+,+}$ & $2_{0,0}$ & $1_{+,+}$ & $1_{+,-}$ & $1_{+,+}$ & $1_{+,+}$ \\
\hline$U(1)$ & 6 & 12 & 4 & 13 & 13 & -5 & -5 & -8 & 4 & -16 & 0 \\
\hline
\end{tabular}

Table 1. The $\mathrm{SU}(5) \times D_{4}$ representations and $\mathrm{U}(1)$ charges of the matter, $\mathrm{RH}$ neutrinos and Higgs superfields.

\begin{tabular}{l||l|l|l|l|l||l|l|l|l}
\hline Flavons & $\xi_{1}$ & $\xi_{2}$ & $\xi_{3}$ & $\xi_{4}$ & $\xi_{5}$ & $\phi$ & $\varphi$ & $\Omega$ & $\Phi$ \\
\hline$D_{4}$ & $1_{+,+}$ & $1_{+,+}$ & $1_{+,-}$ & $1_{+,+}$ & $1_{+,-}$ & $1_{+,-}$ & $1_{+,-}$ & $2_{0,0}$ & $2_{0,0}$ \\
\hline$U(1)$ & -4 & -10 & -2 & -16 & -8 & -9 & -14 & -21 & -9 \\
\hline
\end{tabular}

Table 2. The $D_{4} \times \mathrm{U}(1)$ quantum numbers of the flavons used in the quark and charged lepton sectors.

\begin{tabular}{l||l|l|l|l|l}
\hline Flavons & $\rho_{1}$ & $\rho_{2}$ & $\rho_{3}$ & $\digamma$ & $\Gamma$ \\
\hline$D_{4}$ & $1_{+,+}$ & $1_{+,-}$ & $1_{-,-}$ & $2_{0,0}$ & $2_{0,0}$ \\
\hline$U(1)$ & 10 & 10 & 10 & 10 & 10 \\
\hline
\end{tabular}

Table 3. The $D_{4} \times \mathrm{U}(1)$ quantum numbers of the flavons used in the neutrino sector.

charm quark masses are derived from higher dimensional couplings involving five flavon fields denoted as $\xi_{i=1, \ldots, 5}$. In the down quark and charged lepton sector, four flavon fields denoted as $\phi, \varphi, \Omega$ and $\Phi$ are needed for $D_{4} \times \mathrm{U}(1)$ invariance. When these flavons acquire their VEVs, they break the $D_{4}$ group and lead to appropriate mass matrices of down quarks and charged leptons. In the neutrino sector, five flavons are required for $\mathrm{U}(1)$ invariance. Three of them, denoted as $\rho_{1}, \rho_{2}$ and $\rho_{3}$, are assigned into different $D_{4}$ singlets while the remaining two denoted as $\digamma$ and $\Gamma$ are transforming as $D_{4}$ doublets. The flavons $\rho_{1}$ and $\digamma$ lead to the popular tribimaximal mixing matrix [75], while $\rho_{2}, \rho_{3}$ and $\Gamma$ are responsible for the deviation of the neutrino mixing angles from their TBM values. The quantum numbers under $D_{4} \times \mathrm{U}(1)$ of these five flavons is as depicted in table 3 .

\section{Charged fermion sector}

To derive the Yukawa matrices of the charged fermion sector, we start by the up-type quarks Yukawa matrix which descend from the trilinear interaction terms $10_{i} \cdot 10_{j} \cdot 5_{H_{u}} \equiv T_{i} T_{j} H_{5}$ where $i, j=1,2,3$. However, the up-type quarks Yukawa matrix is generated within our construction from higher order operators derived from several renormalizable terms involving messenger fields $Y_{i}$ and gauge singlet flavon fields $\xi_{i}$, see appendix A for more details on $Y_{i}$ and tables 1 and 2 to check the invariance under $\mathrm{SU}(5) \times D_{4} \times \mathrm{U}(1)$ symmetry. After integrating out these messenger fields ${ }^{7}$ we obtain the invariant effective superpotential

\footnotetext{
${ }^{7}$ The renormalizable superpotentials for the quarks before integrating out the messenger fields are given in appendix A.
} 
for the up quarks

$$
\begin{aligned}
W_{u p}= & \frac{y_{11}^{u}}{\Lambda} T_{1} T_{1} H_{5} \xi_{1}+\frac{y_{12}^{u}}{\Lambda} T_{1} T_{2} H_{5} \xi_{2}+\frac{y_{13}^{u}}{\Lambda} T_{1} T_{3} H_{5} \xi_{3}+\frac{y_{22}^{u}}{\Lambda} T_{2} T_{2} H_{5} \xi_{4} \\
& +\frac{y_{23}^{u}}{\Lambda} T_{2} T_{3} H_{5} \xi_{5}+y_{33}^{u} T_{3} T_{3} H_{5}
\end{aligned}
$$

where $y_{i j}^{u}$ are the Yukawa coupling constants and $\Lambda$ is the cutoff scale of the model which we take as the GUT scale. The $D_{4}$ flavor symmetry is broken by the VEVs of the flavon fields as $\left\langle\xi_{i}\right\rangle=v_{\xi_{i}}$ with $i=1, \ldots, 5$ while the electroweak doublet $H_{u}$ contained in $H_{5}$ acquire its VEV as usual $\left\langle H_{5}\right\rangle=v_{u}$. Assuming that the parameters in $W_{u p}$ are all real, the Yukawa matrix of up-type quarks can be written as

$$
\mathcal{Y}_{u p}=\left(\begin{array}{ccc}
\frac{y_{11}^{u}}{\Lambda} v_{\xi_{1}} & \frac{y_{12}^{u}}{\Lambda} v_{\xi_{2}} & \frac{y_{13}^{u}}{\Lambda} v_{\xi_{3}} \\
\frac{y_{12}^{u}}{\Lambda} v_{\xi_{2}} & \frac{y_{22}^{u}}{\Lambda} v_{\xi_{4}} & \frac{y_{23}^{u}}{\Lambda} v_{\xi_{5}} \\
\frac{y_{13}^{u}}{\Lambda} v_{\xi_{3}} & \frac{y_{23}^{u}}{\Lambda} v_{\xi_{5}} & y_{33}^{u}
\end{array}\right)=\left(\begin{array}{ccc}
a_{11} & a_{12} & a_{13} \\
a_{12} & a_{22} & a_{23} \\
a_{13} & a_{23} & a_{33}
\end{array}\right)
$$

We will now proceed with the down-type quarks and charged leptons generated from the same Yukawa coupling $10_{i} \cdot \overline{5}_{j} \cdot \overline{5}_{H_{d}} \equiv T_{i} F_{j} H_{\overline{5}}$ where $i, j=1,2,3$ are the generation indices. The superpotential leading to the Yukawa matrices is obtained from a renormalizable superpotential that contains messenger fields denoted as $X_{i}$. However, this time we need to add higher-dimensional Higgs representations to differentiate between down quarks and charged lepton masses, in particular we use the adjoint Higgs $H_{24}$ and the 45-dimensional Higgs $H_{\overline{45}}$ for this purpose, see eqs. (2.1) and (2.2). Therefore, by using the superfield assignments in tables 1 and 2 and integrate out the messenger fields, we get the following invariant effective superpotential for the down quarks and charged leptons

$$
W_{e, d}=\frac{y_{11}^{d}}{\left\langle H_{24}\right\rangle^{2}} T_{1} F_{1} \phi \varphi H_{\overline{5}}+\frac{y_{12}^{d}}{\left\langle H_{24}\right\rangle^{2}} T_{1} F_{2,3} \Phi \varphi H_{\overline{5}}+\frac{y_{22}^{d}}{\Lambda^{2}} T_{2} F_{2,3} \Phi H_{24} H_{\overline{45}}+\frac{y_{33}^{d}}{\Lambda} T_{3} F_{2,3} \Omega H_{\overline{5}}
$$

where $y_{i j}^{d}$ are the Yukawa coupling constants associated to the down quarks and charged leptons. To illustrate how the adjoint Higgs contributes to the entries of the Yukawa matrices and leading subsequently to a particular CG factors that distinguish the down quarks Yukawa couplings from those of the charged leptons, let us discuss the $y_{11}^{d}$ effective operator in (3.3). This term is achieved by integrating out the heavy messenger fields from the following renormalizable terms ${ }^{8}$

$$
W_{e, d}^{R e n} \supset F_{1} \phi X_{1}+\bar{X}_{1} \varphi X_{2}+\bar{X}_{2} H_{\overline{5}} T_{1}+X_{1} H_{24} \bar{X}_{1}+X_{2} H_{24} \bar{X}_{2}
$$

After integrating out $X_{1,2}$ and $\bar{X}_{1,2}$ from the first three terms in (3.4), we are left with the first operator in (3.3); $T_{1} F_{1} \phi \varphi H_{\overline{5}}$. On the other hand, the last two terms in eq. (3.4) are responsible for the appearance of the square of the Higgs adjoint VEV $\left\langle H_{24}\right\rangle^{2}$ in the denominator of the effective operator. Specifically, the masses of the messenger pairs $X_{1,2}$ and $\bar{X}_{1,2}$ are achieved when $H_{24}$ acquire its $\mathrm{VEV}^{9}\left\langle H_{24}\right\rangle$ with the group structure given in

\footnotetext{
${ }^{8}$ The coupling constants are omitted in $W_{e, d}^{R e n}$ for clarity.

${ }^{9}$ We assume for simplicity that the VEV of the adjoint Higgs is around the GUT scale as well as the cutoff scale; $\Lambda \equiv\left\langle H_{24}\right\rangle=M_{G U T} \simeq 2 \times 10^{16} \mathrm{GeV}$.
} 
eq. (2.2) which is then followed by integrating out $X_{1} \bar{X}_{1}$ and $X_{2} \bar{X}_{2}$ to obtain eventually the first effective operator in (3.3). According to the group structure of $\left\langle H_{24}\right\rangle$, the down quark mass is multiplied by the inverse of the $\mathrm{CG}$ factors in the first three entries of the adjoint Higgs VEV in eq. (2.2) which is just 1 in this case, while the electron mass is multiplied by the inverse of the fourth and fifth components such that the resulting CG coefficient is $\frac{-2}{3} \times \frac{-2}{3}=\frac{4}{9}$. The same discussion holds for the second effective operator in (3.3) while for the third effective operator the CG factors arise in the numerator. For completeness, when the flavon fields acquire their VEVs in accordance with the following alignment

$$
\langle\phi\rangle=v_{\phi}, \quad\langle\varphi\rangle=v_{\varphi}, \quad\langle\Phi\rangle=\left(v_{\Phi}, 0\right)^{T}, \quad\langle\Omega\rangle=\left(0, v_{\Omega}\right)^{T}
$$

we end up with the Yukawa matrices of the down-type quarks $\mathcal{Y}_{d}$ and charged leptons $\mathcal{Y}_{e}$ expressed as

$$
\begin{aligned}
& \mathcal{Y}_{d}=\left(\begin{array}{ccc}
\frac{y_{11}^{d} v_{\varphi} v_{\phi}}{v_{24}^{2}} & \frac{y_{12}^{d} v_{\varphi} v_{\Phi}}{v_{24}^{2}} & 0 \\
0 & \frac{y_{22}^{d} v_{24} v_{\Phi}}{\Lambda^{2}} & 0 \\
0 & 0 & \frac{y_{33}^{d} v_{\Omega}}{\Lambda}
\end{array}\right)=\left(\begin{array}{ccc}
b_{11} & b_{12} & 0 \\
0 & b_{22} & 0 \\
0 & 0 & b_{33}
\end{array}\right) \\
& \mathcal{Y}_{e}=\left(\begin{array}{ccc}
\frac{4}{9} \frac{y_{11}^{d} v_{\phi}}{v_{24}^{2}} & 0 & 0 \\
\frac{4}{9} \frac{y_{12}^{d} v_{\varphi} v_{\Phi}}{v_{24}^{2}} & \frac{9}{2} \frac{y_{22}^{d} v_{24} v_{\Phi}}{\Lambda^{2}} & 0 \\
0 & 0 & \frac{y_{33}^{d} v_{\Omega}}{\Lambda}
\end{array}\right)=\left(\begin{array}{ccc}
\frac{4}{9} b_{11} & 0 & 0 \\
\frac{4}{9} b_{12} & \frac{9}{2} b_{22} & 0 \\
0 & 0 & b_{33}
\end{array}\right)
\end{aligned}
$$

These Yukawa matrices imply diagonal Yukawa couplings $y_{d}=b_{11}, y_{s}=b_{22}, y_{b}=b_{33}$, $y_{e}=\frac{4}{9} b_{11}, y_{\mu}=\frac{9}{2} b_{22}$ and $y_{\tau}=b_{33}$ where $y_{d}, y_{s}$, and $y_{b}$ stand for the eigenvalues of the down quark Yukawa matrix $\mathcal{Y}_{d}$, and $y_{e}, y_{\mu}$, and $y_{\tau}$ stand for the eigenvalues of the charged lepton Yukawa matrix $\mathcal{Y}_{e}$. Thus, we find that for the third family Yukawa coupling we have the well-known $b-\tau$ unification; $y_{\tau}=y_{b}$ which is still compatible with experimental constraints [33], while for the first two families — instead of the GJ relation — we find alternative GUT predictions with modified CG factors given as

$$
\frac{y_{e}}{y_{d}}=\frac{4}{9}, \quad \frac{y_{\mu}}{y_{s}}=\frac{9}{2}
$$

In general, to test the validity of the GUT Yukawa couplings in a model of low-energy SUSY such as the MSSM, an accurate incorporation of SUSY threshold effects is necessary especially in the case of large or medium $\tan \beta$ [76-79]. However, there have been some studies showing that the threshold corrections may be ignored when the running of fermion masses to the GUT scale are included, see, e.g., [80, 81]. Moreover, to check the validity of the ratios in eq. (3.8), there are two particular constraints for their GUT values developed in reference [62]; these are given by

$$
\frac{\left(1+\bar{\eta}_{l}\right) y_{e}}{\left(1+\bar{\eta}_{q}\right) y_{d}} \approx 0.41_{-0.06}^{+0.02}, \quad \frac{\left(1+\bar{\eta}_{l}\right) y_{\mu}}{\left(1+\bar{\eta}_{q}\right) y_{s}} \approx 4.36 \pm 0.23
$$

where $\bar{\eta}_{l}$ and $\bar{\eta}_{q}$ denote the threshold correction parameters while the numerical values represent the $1 \sigma$ uncertainties. From these relations, one can derive the following double 
ratio at the GUT scale independent of the threshold corrections [62]

$$
\frac{y_{\mu}}{y_{s}} \frac{y_{d}}{y_{e}} \approx 10.7_{-0.8}^{+1.8}
$$

As a result, we find in the present model that the ratios between Yukawa couplings of the first two generations given in eq. (3.8) give rise to the relation $\frac{y_{\mu}}{y_{s}} \frac{y_{d}}{y_{e}} \simeq 10.12$ which is consistent with the double ratio at the GUT scale given in eq. (3.10).

\section{- Comments on proton decay}

Before we turn to the neutrino sector, we give brief comments on proton decay which is one of the most important predictions in GUTs. It is well known that, in the framework of the minimal SUSY SU(5), the fast proton decay comes from the contributions of the dimension four TFF and dimension five TTTF baryon number violating operators. ${ }^{10}$ These operators lead to proton lifetime lower than the limit provided by the Super-Kamiokande experiment [83]. It has been shown that the $d=4$ operators can be prevented by imposing the usual $R$ symmetry like in the case of the MSSM [84]. On the other hand, the $d=5$ operators $T_{i} T_{j} T_{k} F_{l}$ are generically induced via the exchange of color triplet Higgsino [85, 86]. This issue of Higgsino-mediated proton decay ${ }^{11}$ is intimately connected with the so-called doublet-triplet splitting problem - that is the problem of differentiating between the masses of the Higgs triplets and the Higgs doublets contained in the five dimensional Higgs of the SU(5) GUT - The most effective ways proposed in the literature to resolve this splitting problem is provided by the missing partner (MP) and the double missing partner (DMP) mechanisms [90-93].

In our model, the renormalizable operators $T_{i} F_{j} F_{k}$ are forbidden because they transform nontrivially under the $\mathrm{U}(1)$ symmetry and thus, the proton stability at dimension 4 is guaranteed. Moreover, the $d=4$ operators that may arise from the coupling with the flavon fields present in the model are also prevented by the U(1) symmetry as can be checked easily from the field assignments in tables $1-3$.

For the $d=5$ operators, recall first that the usual tree-level Yukawa couplings of the first and second generations are prevented by the U(1) symmetry, and thus the usual operators $T_{i} T_{j} T_{k} F_{l}$ are absent in our model. On the other hand, since the masses of quarks and charged leptons are generated from the effective operators in eqs. (3.1) and (3.3) that involve flavon fields, the operator TTTF inducing proton decay can arise - after integrating out the color-triplet Higgs with GUT-scale mass $M_{T}$ - from higher dimension-7 and dimension- 8 operators of the form

$$
\frac{1}{M_{T}} T_{i} T_{j} T_{k} F_{l} \frac{f_{1}}{M} \frac{f_{2}}{M}\left(\frac{f_{3}}{M}\right)^{n}
$$

where $M$ stands for the cutoff scale $\Lambda$ or the VEV of the adjoint Higgs, $n=0,1$ and $f_{i}$ stands for the flavon fields $\xi_{i=1, \ldots, 5}, \phi, \varphi, \Phi$, and $\Omega$. It is clear that these higher dimensional

\footnotetext{
${ }^{10}$ For a brief review on proton decay coming from dimension four and five operators, see for example the appendix C of ref. [44].

${ }^{11}$ The proton decay via dimensional five operators is mediated by the heavy color triplet Higgsino, and is obtained after integrating out the colored Higgs triplet, for more details see for example refs. [87-89].
} 
operators are consistent with the messenger content given in appendix A considering that they are derived from the effective operators in eqs. (3.1) and (3.3) which are themselves obtained after integrating out a set of messenger fields required to make the model renormalizable. As an illustration, after integrating out the messenger fields $Y_{1}$ and $\bar{Y}_{1}$ from the renormalizable superpotential $W_{u p}^{R e n} \supset H_{5} T_{1} Y_{1}+\bar{Y}_{1} T_{1} \xi_{1}$ (see appendix A for the complete superpotential), we obtain the first operator in eq. (3.1) given by $(1 / \Lambda) T_{1} T_{1} H_{5} \xi_{1}$. Then, after integrating out the colored Higgs triplet from this resulting operator and the last operator in eq. (3.3), we obtain the dimension-7 operator

$$
\frac{1}{M_{T}} \frac{1}{\Lambda^{2}} T_{1} T_{1} T_{3} F_{2,3} \xi_{1} \Omega
$$

Since $M_{T}$ and $\Lambda$ are both expected to be at the GUT scale, it is straightforward to realize that the contribution of the operator (3.12) to proton decay is sufficiently suppressed. The same discussion holds for all the allowed $T_{i} T_{j} T_{k} F_{l}$ operators generated with a highly suppressed factors manifested by the ratios $\frac{v_{f_{1}} v_{f_{2}}}{M_{T} \Lambda^{2}}$ for dimension- 7 operators and $\frac{v_{f_{1}} v_{f_{2}} v_{f_{3}}}{M_{T} \Lambda^{3}}$ for dimension- 8 operators.

\section{Neutrino sector}

The fermion sector of the supersymmetric SU(5)-GUT model is extended by three righthanded neutrino superfields $N_{i=1,2,3}^{c}$ transforming as gauge singlets $1_{i}$, and carrying quantum numbers under the $D_{4} \times \mathrm{U}(1)$ flavor group. Therefore, the light active neutrino masses are generated through the famous type I seesaw mechanism. In our setup with the quantum numbers of matter and Higgs superfields given in table 1, five flavon superfields are required for $\mathrm{U}(1)$ invariance in such a way that they all couple only to the Majorana mass term $N_{i}^{c} N_{j}^{c}$ and they all carry the same $\mathrm{U}(1)$ charge $q_{\mathrm{U}(1)}=10$. Three of these flavons denoted as $\rho_{1}, \rho_{2}$ and $\rho_{3}$ are assigned to the $D_{4}$ singlets $1_{+,+}, 1_{+,-}$and $1_{-,-}$respectively, while the remaining two denoted as $\Gamma$ and $\digamma$ are assigned to the $D_{4}$ doublet $2_{0,0}$. Thus, by using the quantum numbers in tables 1 and 2, the superpotential invariant under the $\mathrm{SU}(5) \times D_{4} \times \mathrm{U}(1)$ group is given by

$$
\begin{aligned}
\mathcal{W}_{\nu}= & \lambda_{1} N_{1}^{c} F_{1} H_{5}+\lambda_{2} N_{3,2}^{c} F_{2,3} H_{5}+\lambda_{3} N_{1}^{c} N_{1}^{c} \rho_{1}+\lambda_{4} N_{3,2}^{c} N_{3,2}^{c} \rho_{1} \\
& +\lambda_{5} N_{1}^{c} N_{3,2}^{c} \digamma+\lambda_{6} N_{1}^{c} N_{3,2}^{c} \Gamma+\lambda_{7} N_{3,2}^{c} N_{3,2}^{c} \rho_{2}+\lambda_{8} N_{3,2}^{c} N_{3,2}^{c} \rho_{3}
\end{aligned}
$$

where $\lambda_{i=1, \ldots, 8}$ are Yukawa coupling constants. The first two terms in $\mathcal{W}_{\nu}$ are the Dirac Yukawa terms leading to the Dirac mass matrix $m_{D}$ while the remaining couplings give rise to the Majorana mass matrix $m_{M}$. Our aim here is to achieve a configuration from $\mathcal{W}_{\nu}$ that is consistent with the well-known trimaximal mixing matrix which allows naturally for nonzero reactor angle $\theta_{13}$, nonmaximal atmospheric angle $\theta_{23}$ and for $\sin ^{2} \theta_{12} \neq 1 / 3$. The $T M_{2}$ matrix is known to preserve the second column of the famous TBM matrix which is ruled out by the data from reactor neutrino experiments; nevertheless, since it is congruous with the solar and atmospheric angles, it can still be used as a good zeroth-order approximation. Before we develop our neutrino mass matrix $m_{\nu}$, let us recall briefly some of the properties of the mass matrix acquired by TBM and $T M_{2}$. For the TBM matrix, the 
mass matrix $m_{\nu}$ must respects the well-known $\mu-\tau$ symmetry referring to the invariance of $m_{\nu}$ after the interchange of the $\mu$ and $\tau$ indices [51-55], and the following condition among the entries of $m_{\nu}:\left(m_{\nu}\right)_{11}+\left(m_{\nu}\right)_{12}=\left(m_{\nu}\right)_{22}+\left(m_{\nu}\right)_{23}$. The deviation from TBM is realized by adding small perturbations to $m_{\nu}$ in such a way that the $\mu-\tau$ symmetry gets broken. There are in particular two matrix perturbations that give rise to a mass matrix with magic symmetry known to be consistent with $T M_{2}$ [63]; these two matrices are given as follows

$$
\delta m_{\nu}^{1}=\left(\begin{array}{ccc}
0 & 0 & \mathrm{k} \\
0 & \mathrm{k} & 0 \\
\mathrm{k} & 0 & 0
\end{array}\right), \quad \delta m_{\nu}^{2}=\left(\begin{array}{ccc}
0 & \mathrm{k} & 0 \\
\mathrm{k} & 0 & 0 \\
0 & 0 & \mathrm{k}
\end{array}\right)
$$

Now, let us use these properties in our superpotential $\mathcal{W}_{\nu}$ and derive the mass matrices of Dirac and Majorana neutrinos to calculate the total neutrino mass matrix using the type I seesaw formula $m_{\nu}=m_{D} m_{M}^{-1} m_{D}^{T}$. The Higgs doublet develops its VEV as usual $\left\langle H_{u}\right\rangle=v_{u}$ while we assume that the VEVs of the $D_{4}$ breaking flavon fields point in the following directions

$$
\left\langle\rho_{1}\right\rangle=v_{\rho_{1}}, \quad\left\langle\rho_{2}\right\rangle=v_{\rho_{2}}, \quad\left\langle\rho_{3}\right\rangle=v_{\rho_{3}}, \quad\langle\digamma\rangle=\left(v_{\digamma}, v_{\digamma}\right)^{T}, \quad\langle\Gamma\rangle=\left(0, v_{\Gamma}\right)^{T}
$$

The study of the potential which gives rise to the alignment of the flavon doublets is discussed in appendix D. By using the tensor product of $D_{4}$ irreducible representations given in eqs. (C.2) and (C.3), we find that the Dirac and Majorana mass matrices have the following forms

$$
\begin{aligned}
m_{D} & =v_{u}\left(\begin{array}{ccc}
\lambda_{1} & 0 & 0 \\
0 & \lambda_{2} & 0 \\
0 & 0 & \lambda_{2}
\end{array}\right), \quad m_{M}=m_{M_{1}}+m_{M_{2}} \\
\text { with } m_{M} & =\left(\begin{array}{ccc}
\lambda_{3} v_{\rho_{1}} & \lambda_{5} v_{\digamma} & \lambda_{5} v_{\digamma} \\
\lambda_{5} v_{\digamma} & 0 & 2 \lambda_{4} v_{\rho_{1}} \\
\lambda_{5} v_{\digamma} & 2 \lambda_{4} v_{\rho_{1}} & 0
\end{array}\right)+\left(\begin{array}{ccc}
0 & 0 & \lambda_{6} v_{\Gamma} \\
0 & \lambda_{7} v_{\rho_{2}}-\lambda_{8} v_{\rho_{3}} & 0 \\
\lambda_{6} v_{\Gamma} & 0 & \lambda_{7} v_{\rho_{2}}+\lambda_{8} v_{\rho_{3}}
\end{array}\right)
\end{aligned}
$$

The Majorana mass matrix is decomposed in terms of two matrices to show that the TBM conditions and its deviation to the $T M_{2}$ are obtained from $m_{M_{1}}$ and $m_{M_{2}}$, respectively. Accordingly, the $\mu-\tau$ symmetry and the condition $\left(m_{\nu}\right)_{11}+\left(m_{\nu}\right)_{12}=\left(m_{\nu}\right)_{22}+\left(m_{\nu}\right)_{23}$ require the imposition of the following assumptions on $m_{D}$ and $m_{M_{1}}$

$$
\lambda_{1}=\lambda_{2} \quad \text { and } \quad \lambda_{3} v_{\rho_{1}}+\lambda_{5} v_{\digamma}=2 \lambda_{4} v_{\rho_{1}}
$$

while the deviation from TBM to $T M_{2}$ requires a mass matrix with the magic symmetry which is conceivable by the imposition of the following assumption on $m_{M_{2}}$

$$
\lambda_{8} v_{\rho_{3}}=-\lambda_{7} v_{\rho_{2}}=\lambda_{6} v_{\Gamma} / 2
$$

leading to the form of the matrix perturbation $\delta m_{\nu}^{1}$ in (4.2). The plausibility of these assumptions is discussed in appendix D. To simplify the parametrization of the total neutrino 
mass matrix and later the expressions of neutrino masses as well as the mixing angles, we parametrize the Majorana mass matrix as follows

$$
m_{M}=M_{R}\left(\begin{array}{ccc}
a & b & b+\mathrm{k} \\
b & \mathrm{k} & c \\
b+\mathrm{k} & c & 0
\end{array}\right)
$$

where $M_{R}$ is the mass scale of the heavy RH Majorana neutrinos and $a=\frac{\lambda_{3} v_{\rho_{1}}}{M_{R}}, b=\frac{\lambda_{5} v_{\digamma}}{M_{R}}$, $c=\frac{2 \lambda_{4} v_{\rho_{1}}}{M_{R}}$ and $\mathrm{k}=\frac{\lambda_{6} v_{\Gamma}}{M_{R}}$. The usual canonical seesaw formula $m_{\nu}=m_{D} m_{M}^{-1} m_{D}^{T}$ yields the total neutrino mass matrix

$$
m_{\nu}=\frac{m_{0}}{P}\left(\begin{array}{ccc}
-(a+b)^{2} & (a+b)(b+\mathrm{k}) & b^{2}-\mathrm{k}^{2}-b(\mathrm{k}-a) \\
(a+b)(b+\mathrm{k}) & -(b+\mathrm{k})^{2} & -a^{2}-a b+b^{2}+\mathrm{k} b \\
b^{2}-\mathrm{k}^{2}-b(\mathrm{k}-a) & -a^{2}-a b+b^{2}+\mathrm{k} b & a \mathrm{k}-b^{2}
\end{array}\right)
$$

where $m_{0}=\frac{\left(\lambda_{1} v_{u}\right)^{2}}{M_{R}}$ and $P=(a+2 b+\mathrm{k})\left(a \mathrm{k}-a^{2}+b^{2}-\mathrm{k}^{2}\right)$. It is clear to verify that in the limit where $\mathrm{k} \rightarrow 0$, this matrix obeys the $\mu-\tau$ symmetry, and thus it is diagonalized by the TBM matrix which is $C P$ conserving and predicts $\theta_{13}=0$ and $\theta_{23}=\pi / 4$. Therefore, the presence of $\mathrm{k}$ is necessary to break the $\mu-\tau$ symmetry and produce a small deviation from the TBM pattern as mentioned above. Without loss of generality, only the parameter $\mathrm{k}$ is taken to be complex - $\mathrm{k} \rightarrow|\mathrm{k}| e^{i \phi_{k}}$ where $\phi_{k}$ is a $C P$ violating phase - which is sufficient to ensure $C P$ violation in the lepton sector. On the other hand, the matrix $m_{\nu}$ enjoys the magic symmetry property which refers to the equality of the sum of each row and the sum of each column in the neutrino mass matrix. This property implies that the neutrino matrix is diagonalized by the well-known trimaximal mixing matrix $\mathcal{U}_{T M_{2}}$ so that $m_{\nu}^{\text {diag }}=\mathcal{U}_{T M_{2}}^{\dagger} m_{\nu} \mathcal{U}_{T M_{2}}$ with

$$
\mathcal{U}_{T M_{2}}=\left(\begin{array}{ccc}
\sqrt{\frac{2}{3}} \cos \theta & \frac{1}{\sqrt{3}} & \sqrt{\frac{2}{3}} \sin \theta e^{-i \sigma} \\
-\frac{\cos \theta}{\sqrt{6}}-\frac{\sin \theta}{\sqrt{2}} e^{i \sigma} & \frac{1}{\sqrt{3}} & \frac{\cos \theta}{\sqrt{2}}-\frac{\sin \theta}{\sqrt{6}} e^{-i \sigma} \\
-\frac{\cos \theta}{\sqrt{6}}+\frac{\sin \theta}{\sqrt{2}} e^{i \sigma} & \frac{1}{\sqrt{3}} & -\frac{\cos \theta}{\sqrt{2}}-\frac{\sin \theta}{\sqrt{6}} e^{-i \sigma}
\end{array}\right)
$$

The full mixing matrix is given by $\mathcal{U}_{\nu}=\mathcal{U}_{T M_{2}} \mathcal{U}_{P}$ where $\mathcal{U}_{P}=\operatorname{diag}\left(1, e^{i \frac{\alpha_{21}}{2}}, e^{i \frac{\alpha_{31}}{2}}\right)$ is a diagonal matrix that contains the Majorana phases $\alpha_{21}$ and $\alpha_{31}$. The parameters $\theta$ and $\sigma$ are respectively an arbitrary angle and a phase which will be related to the neutrino oscillation parameters; the observed neutrino mixing angles $\theta_{i j}$ and the Dirac $C P$ phase $\delta_{C P}$. The diagonalization of the neutrino matrix (4.8) by $\mathcal{U}_{T M_{2}}$ induces relations between our model parameters and the trimaximal mixing parameters $\sigma$ and $\theta$, we find

$$
\tan 2 \theta=\frac{\sqrt{3}|\mathrm{k}| \sqrt{b^{2} \cos ^{2} \phi_{\mathrm{k}}+a^{2} \sin ^{2} \phi_{k}}}{2 a b-b|\mathrm{k}| \cos \phi_{k}}, \quad \tan \sigma=\frac{-a}{b} \tan \phi_{k}
$$

As a result, the eigenmasses of $m_{\nu}$ are as follows

$$
\begin{aligned}
\left|m_{1}\right| & =\frac{m_{0}}{\sqrt{(a-b)^{2}-|\mathrm{k}|(a-b) \cos \phi_{k}+\left(|\mathrm{k}|^{2} / 4\right)}}, \quad\left|m_{2}\right|=\frac{m_{0}}{\sqrt{(a+2 b)^{2}+2|\mathrm{k}|(a+2 b) \cos \phi_{k}+|\mathrm{k}|^{2}}} \\
\left|m_{3}\right| & =\frac{m_{0}}{\sqrt{(a+b)^{2}-|\mathrm{k}|(a+b) \cos \phi_{k}+\left(|\mathrm{k}|^{2} / 4\right)}}
\end{aligned}
$$


where the denominators of these masses corresponds to ratios of the right-handed neutrino masses and their mass scale $M_{R}$

$$
\begin{aligned}
& \frac{\left|M_{1}\right|}{M_{R}}=\sqrt{(a-b)^{2}-|\mathrm{k}|(a-b) \cos \phi_{k}+\left(|\mathrm{k}|^{2} / 4\right)} \\
& \frac{\left|M_{2}\right|}{M_{R}}=\sqrt{(a+2 b)^{2}+2|\mathrm{k}|(a+2 b) \cos \phi_{k}+|\mathrm{k}|^{2}} \\
& \frac{\left|M_{3}\right|}{M_{R}}=\sqrt{(a+b)^{2}-|\mathrm{k}|(a+b) \cos \phi_{k}+\left(|\mathrm{k}|^{2} / 4\right)}
\end{aligned}
$$

Regarding the mixing angles, it is well-known that the total lepton mixing matrix is derived from the product between two matrices; $U_{P M N S}=\mathcal{U}_{l}^{\dagger} \mathcal{U}_{\nu}$ where $\mathcal{U}_{l}$ is the matrix that diagonalizes the charged lepton mass matrix while $\mathcal{U}_{T M_{2}}$ is as described above. In the

present model however, it is easy to check that the charged lepton mixing angles $\theta_{i j}^{l}$ derived from the diagonalization of $m_{l}=v_{d} \mathcal{Y}_{e}$ - where $\mathcal{Y}_{e}$ is given in eq. (3.7) - are all equals to zero; thus, they do not affect the neutrino mixing angles derived from $\mathcal{U}_{\nu}$. Therefore, by using the PDG standard parametrization of the PMNS matrix [94], the reactor, solar and atmospheric angles are expressed as

$$
\sin ^{2} \theta_{13}=\frac{2}{3} \sin ^{2} \theta, \sin ^{2} \theta_{12}=\frac{1}{3-2 \sin ^{2} \theta}, \sin ^{2} \theta_{23}=\frac{1}{2}-\frac{3 \sin 2 \theta}{2 \sqrt{3}\left(3-2 \sin ^{2} \theta\right)} \cos \sigma .
$$

Before we perform a numerical analysis of neutrino masses and model parameters, we should notice that the above masses and mixing parameters are valid at the GUT scale. Therefore, to match these parameters with the experimental values (of the mixing angles, the $C P$ phase and the mass squared differences), their evolution from the GUT scale to low energy must be carried out. However, although the final values are model dependent, it was illustrated in ref. [82] that for SUSY models, if $\tan \beta$ is small, the RG-induced effects on the above parameters are controllable and can be safely neglected. Accordingly, since the type I seesaw mechanism is related to physics at very high energy scales, we can work in a scenario with small neutrino Yukawa couplings in such a way that their contribution to the RG evolution can be neglected [62].

\section{$5 \quad$ Numerical analysis and results}

In this section, we carry out a detailed numerical analysis for both charged fermion and neutrino sectors. For the charged fermion sector, we fix the values of the model parameters in order to reproduce the observed fermion Yukawa couplings and the CKM mixing parameters at the GUT scale within $1 \sigma$ ranges. As for the neutrino sector, we constrain our model parameters using the $3 \sigma$ allowed range of the neutrino oscillation parameters. We also use constraints from non-oscillatory experiments to make predictions concerning the physical observables $m_{\beta \beta}, m_{\beta}$, and $\sum m_{i}$.

\subsection{Numerical fits for charged fermion sector}

Our model predicts the Yukawa couplings and mixing parameters at the GUT scale which we assumed to be also the flavor symmetry breaking scale. To compare the obtained 


\begin{tabular}{|l|l||l|l|}
\hline Model parameters & Values & Model parameters & Values \\
\hline$a_{11}$ & $0.1189 \times 10^{-4}$ & $b_{11}$ & $0.44929 \times 10^{-4}$ \\
\hline$a_{12}$ & $0.1530 \times 10^{-2}$ & $\left|b_{12}\right|$ & $0.240406 \times 10^{-3}$ \\
\hline$a_{13}$ & $0.2487 \times 10^{-2}$ & $b_{22}$ & $0.9545 \times 10^{-2}$ \\
\hline$a_{22}$ & $0.2076 \times 10^{-2}$ & $b_{33}$ & 0.0705 \\
\hline$a_{23}$ & 0.01857 & $\epsilon$ & $\frac{\pi}{3}$ \\
\hline$a_{33}$ & 0.5218 & \multicolumn{2}{|l}{} \\
\cline { 1 - 2 }
\end{tabular}

Table 4. Parameters of the quark and charged leptons Yukawa matrices at the GUT scale with $\tan \beta=10$.

spectrum of our model with the data extrapolated at the GUT scale, the experimental values must run up to the GUT scale taking into account the SUSY parameters; $\tan \beta$ and SUSY threshold correction effects. Such an analysis has been performed in ref. [62], where the extracted CKM parameters and all Yukawa couplings at the GUT scale for $\tan \beta=5$ and $\tan \beta=10$ with $M_{S U S Y}=1 \mathrm{TeV}$ and $\eta_{b}=-0.2437$ are given in table 3 of ref. [41]. In our model, recall that the mass matrices of the charged fermions are generally expressed as

$$
m_{u p}=v_{u} \mathcal{Y}_{u p}, \quad m_{\text {down }}=v_{d} \mathcal{Y}_{d}, \quad m_{\text {lep }}=v_{d} \mathcal{Y}_{e}
$$

where $\mathcal{Y}_{u p}, \mathcal{Y}_{d}$ and $\mathcal{Y}_{e}$ refer to the Yukawa matrices of up quarks, down quarks and charged leptons obtained in eqs. (3.2), (3.6) and (3.7), respectively. Recall also that the ratio between the Higgs VEVs $v_{u}$ and $v_{d}$ is defined as $\tan \beta=\frac{v_{u}}{v_{d}}$ while the SM Higgs VEV is $v=\sqrt{v_{u}^{2}+v_{d}^{2}}=174 \mathrm{GeV}$, we have

$$
m_{u p}=v \sin \beta \mathcal{Y}_{u p}, \quad m_{\text {down }}=v \cos \beta \mathcal{Y}_{d}, \quad m_{\text {lep }}=v \cos \beta \mathcal{Y}_{e}
$$

We assume in our calculations that all Yukawa coupling constants are real except for $y_{16}$ which is complex and leads to the complex nature of the parameter $b_{12}=\left|b_{12}\right| e^{i \epsilon}$, and subsequently to the $C P$ violation in the quark sector. Moreover, the Yukawa matrices in eqs. (3.2) and (3.6) involve the following independent parameters $\left[a_{11}, a_{12}, a_{13}, a_{22}, a_{23}\right.$, $a_{33}, b_{11}, b_{12},\left|b_{22}\right|, b_{33}$ and $\left.\epsilon\right]$, which we need to fix in order to perform our numerical analysis at the GUT scale. Next, we fix these input parameters for two cases: $\tan \beta=10$ and $\tan \beta=5$. For $\tan \beta=10$, the numerical values are reported in table 4 . The values reported in this table are fixed respecting the fact that the magnitude of the flavon VEVs are smaller than the flavor symmetry breaking scale; $v_{\text {flavons }}<M_{G U T}$, while we fix the phase $\epsilon$ to the value $\frac{\pi}{3}$ which yields the correct experimental fit of the $C P$-violating Dirac phase of the quark sector. The above estimates concerning the input parameters produce the values of the physical quantities - namely the quark mixing angles, the Yukawa couplings and the $C P$ phase - at the GUT scale; these numerical values are as reported in table 5 . We repeat the same numerical fit for $\tan \beta=5$, where the input parameters and the output for the physical parameters are reported in tables 6 and 7, respectively. This fit has been performed using the Mixing Parameter Tools package [82]. The obtained values are in a good agreement with the GUT scale data for both $\tan \beta=5$ and $\tan \beta=10$ [41, 62]. 


\begin{tabular}{|l|l||l|l|l|l|}
\hline Observables & values & Observables & values & Observables & values \\
\hline$\theta_{12}^{q} /^{\circ}$ & 13.0433 & $y_{u}$ & $2.88221 \times 10^{-6}$ & $y_{d}$ & $4.33494 \times 10^{-6}$ \\
\hline$\theta_{13}^{q} /^{\circ}$ & 0.18015 & $y_{c}$ & $1.40921 \times 10^{-3}$ & $y_{s}$ & $9.79486 \times 10^{-5}$ \\
\hline$\theta_{23}^{q} /^{\circ}$ & 2.05405 & $y_{t}$ & 0.51988 & $y_{b}$ & $7.01501 \times 10^{-3}$ \\
\hline$\delta_{C P}^{q} /^{\circ}$ & 69.166 & \multicolumn{4}{|l}{} \\
\cline { 1 - 5 }
\end{tabular}

Table 5. The predictions for the Yukawa eigenvalues, the mixing angles and the $C P$ phase of the quark sector for $\tan \beta=10$.

\begin{tabular}{|l|l||l|l|}
\hline Model parameters & Values & Model parameters & Values \\
\hline$a_{11}$ & $0.12397 \times 10^{-4}$ & $b_{11}$ & $0.226238 \times 10^{-4}$ \\
\hline$a_{12}$ & $0.15866 \times 10^{-3}$ & $\left|b_{12}\right|$ & $0.11827 \times 10^{-3}$ \\
\hline$a_{13}$ & $0.25912 \times 10^{-2}$ & $b_{22}$ & $0.47008 \times 10^{-3}$ \\
\hline$a_{22}$ & $0.21459 \times 10^{-2}$ & $b_{33}$ & 0.036597 \\
\hline$a_{23}$ & 0.019344 & $\epsilon$ & $\frac{\pi}{3}$ \\
\hline$a_{33}$ & 0.5435 & & \\
\hline
\end{tabular}

Table 6. Parameters of the quark and charged leptons Yukawa matrices at the GUT scale with $\tan \beta=5$.

\begin{tabular}{|c|c|c|c|c|c|}
\hline Observables & values & Observables & values & Observables & values \\
\hline$\theta_{12} /^{\circ}$ & 13.0295 & $y_{u}$ & $2.92027 \times 10^{-6}$ & $y_{d}$ & $4.16836 \times 10^{-6}$ \\
\hline$\theta_{13} /^{\circ}$ & 0.18019 & $y_{c}$ & $1.42946 \times 10^{-3}$ & $y_{s}$ & $9.21045 \times 10^{-5}$ \\
\hline$\theta_{23} /^{\circ}$ & 2.05418 & $y_{t}$ & 0.53331 & $y_{b}$ & $6.95358 \times 10^{-3}$ \\
\hline$\delta_{C P}^{q} /{ }^{\circ}$ & 69.1801 & & & & \\
\hline
\end{tabular}

Table 7. The predictions for the Yukawa eigenvalues, the mixing angles and the $C P$ phase of the quark sector for $\tan \beta=5$.

\subsection{Neutrino phenomenology}

The fact that the neutrino mass ordering remains unknown requires the investigation of the two possible options: either $\Delta m_{31}^{2}>0$ referred to as normal mass hierarchy or $\Delta m_{32}^{2}<0$ known as the inverted mass Hierarchy (IH). In the latter case that implies $m_{3}<m_{2}<m_{1}$, it is easy to deduce from the first relation in eq. (4.13) as well as the $3 \sigma$ region of the reactor angle from ref. [3] that the parameter $\theta$ lies in the interval $0.1763 \leq \theta \leq 0.1920$. On the other hand, by requiring the values of the mass-squared differences $\Delta m_{i j}^{2}$ within their $3 \sigma$ experimental ranges and using the eigenmasses in eq. (4.11) as well as the constraint on the sum of neutrino masses from cosmological observations $\sum m_{i}<0.12 \mathrm{eV}$ [73], we find that $\theta$ lies in the interval $0.398 \lesssim \theta \lesssim 0.579$ which implies that both $\sin ^{2} \theta_{13}$ and $\sin ^{2} \theta_{12}$ fall far outside their $3 \sigma$ experimental range. For this reason, the IH scheme is excluded in our model. 
As regards to the NH scheme, we rewrite the masses $m_{2}$ and $m_{3}$ in terms of the lightest neutrino mass $m_{1}$ and the mass squared differences as $m_{2}=\sqrt{m_{1}^{2}+\Delta m_{21}^{2}}$ and $m_{3}=$ $\sqrt{m_{1}^{2}+\Delta m_{31}^{2}}$. Moreover, by using eqs. (4.11) and (4.13), these masses can also be expressed as a function of the free parameters $a, b, \mathrm{k}$ and $\phi_{k}$ to which ascribe the smallness of neutrino masses. Thus, we allow $a, b$ and $\mathrm{k}$ to vary in the range $[-1,1]$ while we allow $\phi_{k}$ to vary in the range $[0, \pi]$. In figure 1 , the trimaximal mixing parameters $\sigma$ (top left panel) and $\theta$ (top right panel) are projected on the planes $\left(\sin ^{2} \theta_{23}, \mathrm{k}\right)$ and $\left(\sin ^{2} \theta_{13}, \mathrm{k}\right)$ respectively. As inputs, the angle $\theta$ is allowed to vary in the range $[0, \pi / 2]$ while the phase $\sigma$ is randomly varied in the range $[0,2 \pi]$. From the left panel of figure 1 , we observe from the scattered points that for the atmospheric angle only the lower octant $\left(\sin ^{2} \theta_{23}<0.5\right)$ is allowed. Therefore, an important prediction of the current model is that it excludes the maximal as well as the higher octant of the atmospheric angle. Moreover, the $3 \sigma$ allowed intervals of the oscillation parameters restrict the range of $\sigma$ as well as the range of the parameter $\mathrm{k}$

$$
0.57565 \lesssim \sigma \lesssim 1.57073, \quad-0.52597 \lesssim \mathrm{k} \lesssim 0.55115
$$

Notice by the way that the parameter $\mathrm{k}$ is responsible for the deviation from the TBM values of the mixing angles. This deviation is encoded in the parameter $\theta$ which is easily seen when we set $\theta \rightarrow 0$ in eq. (4.13) resulting to restore the TBM values. From the top right panel of figure 1 , we find that the range of $\theta$ is also restricted to $0.17548 \lesssim \theta \lesssim 0.19129$ while the range of the reactor angle remains almost unchanged compared to its $3 \sigma$ allowed range. In figure 1 , the bottom panel shows the correlation between the parameters $a$ and $b$ with the color code showing the phase $\phi_{k}$. By taking into account the $3 \sigma$ experimental ranges of $\Delta m_{i j}^{2}, \sin \theta_{i j}$ and $\delta_{C P}$ from the most recent global fit by NuFIT collaboration [3], and the current cosmological upper bound on the sum of the three light neutrino masses given by $\sum m_{i}<0.12 \mathrm{eV}$, we find that the range of the phase $\phi_{k}$ gets more restricted compared to its input range; $0.55275 \lesssim \phi_{k} \lesssim 2.56095$. Regarding the Dirac $C P$ phase $\delta_{C P}$, the results reported by the $\mathrm{T} 2 \mathrm{~K}$ long-baseline experiment showed strong hints for $C P$ violation in neutrino oscillations while $C P$ conservation is disfavored at $2 \sigma$ level [6]. One approach to estimate the magnitude of $\delta_{C P}$ is by means of the Jarlskog invariant parameter defined as $J_{C P}=\operatorname{Im}\left(\mathcal{U}_{e 1} \mathcal{U}_{\mu 1}^{*} \mathcal{U}_{\mu 2} \mathcal{U}_{e 2}^{*}\right)$. In the PDG standard parametrization, this parameter is exhibited in terms of the three mixing angles and the Dirac $C P$ phase as follows [94]

$$
J_{C P}=\frac{1}{8} \sin 2 \theta_{12} \sin 2 \theta_{13} \sin 2 \theta_{23} \cos \theta_{13} \sin \delta_{C P}
$$

while in the case of the trimaximal mixing, it takes a simpler form given by $J_{C P}^{T M}=$ $(1 / 6 \sqrt{3}) \sin 2 \theta \sin \sigma$. By matching $J_{C P}^{T M}$ with eq. (5.4), we find a correlation between the Dirac $C P$ phase, the arbitrary phase $\sigma$, and the atmospheric angle

$$
\sin 2 \theta_{23} \sin \delta_{C P}=\sin \sigma
$$

Taking into account the fact that atmospheric angle is well determined experimentally as well as the fact that the range of $\sigma$ given in eq. (5.3) excludes the exact value of $n \pi$ with $n$ can be any integer, it is easy to deduce analytically that the $C P$ conserving values of 

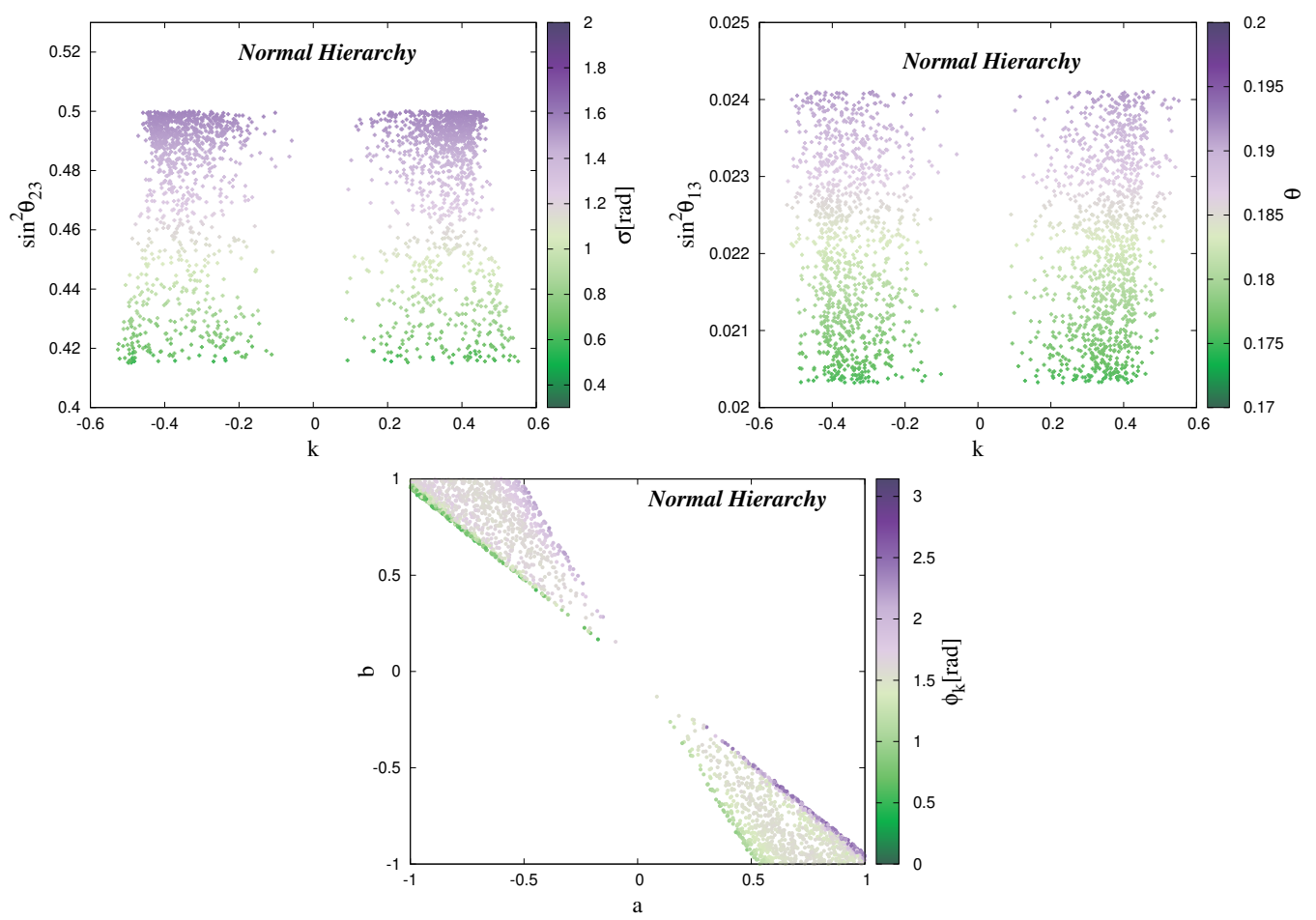

Figure 1. The allowed points of the trimaximal mixing parameters $\sigma$ (top left panel) and $\theta$ (top right panel) projected on the planes $\left(\sin ^{2} \theta_{23}, \mathrm{k}\right)$ and $\left(\sin ^{2} \theta_{13}, \mathrm{k}\right)$ respectively. The bottom panel shows the allowed points in the parameter space of $(a, b)$ with the color code indicating the phase $\phi_{k}$.

$\delta_{C P}$ are not allowed which implies that the present model admits only the $C P$ violating values of $\delta_{C P}$.

- Neutrino masses from non-oscillatory experiments

Constraining the absolute neutrino mass scale is one of the most important purposes of the forthcoming neutrino experiments. This scale can be probed by various non-oscillatory neutrino experiments. Cosmological observations are in particular a powerful tool to probe the total sum of neutrino masses. Indeed, in the framework of $\Lambda$ CDM model with three massive active neutrinos, the latest Planck data combined with baryon acoustic oscillations (BAO) measurements provided an upper bound on the sum of neutrino masses of $\sum m_{i}<$ $0.12 \mathrm{eV}$ [73]; see also ref. [74] for a comprehensive analysis of the changes in the upper bounds of $\sum m_{i}$ after taking into account neutrino oscillation data. Another way to probe this scale is through direct neutrino mass determination where the study of the electron energy spectrum near its endpoint region is up to date the most sensitive method to determine the electron antineutrino mass. The effective electron neutrino mass is defined in terms of the three neutrino mass eigenvalues $m_{i}$ and the flavor mixing parameters $U_{e i}$ as $m_{\beta}=\left(\sum_{i}\left|U_{e i}\right|^{2} m_{i}^{2}\right)^{1 / 2}$. Currently, the most valid bounds on $m_{\beta}$ are presented by the KATRIN experiment which provides an upper limit on the electron antineutrino mass of 1.1 $\mathrm{eV}[95]$ and eventually aims at a sensitivity of $0.2 \mathrm{eV}$ [96]. Using the upper limit $\sum m_{i}<0.12$ $\mathrm{eV}$ and the neutrino oscillation parameters $\left(\theta_{i j}\right.$ and $\left.\Delta m_{i j}^{2}\right)$ within their currently allowed 

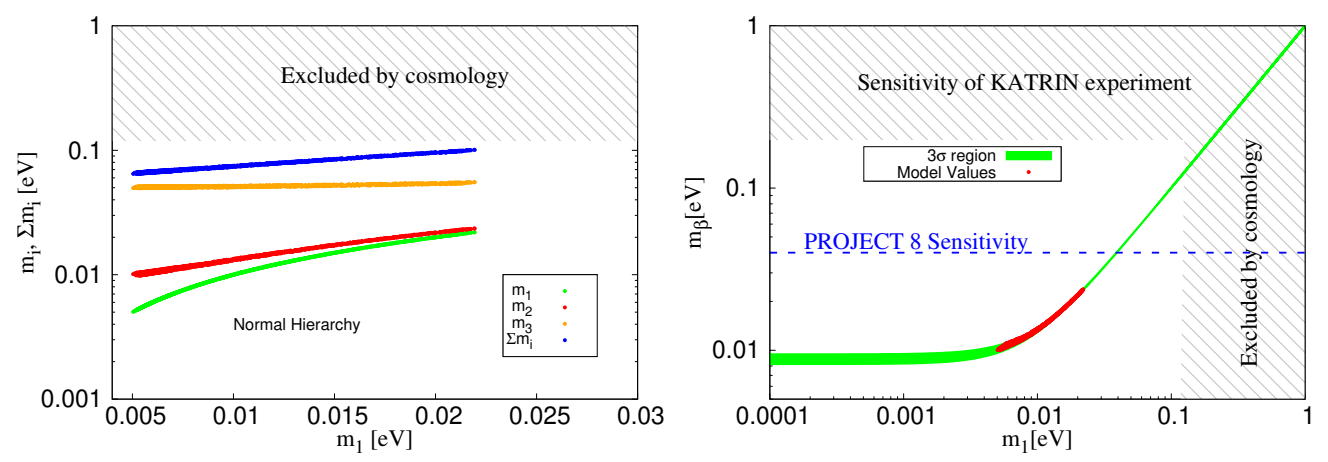

Figure 2. Left: prediction for the absolute neutrino masses and their sum $\sum m_{i}$ as a function of $m_{1}$. Right: $m_{\beta}$ as a function of $m_{1}$ where the gray dashed vertical region is disfavored by Planck $+\mathrm{BAO}$ while the gray dashed horizontal region is the limit on $m_{\beta}$ from KATRIN collaboration.

$3 \sigma$ ranges as well as the restricted interval of our model parameters given in the previous subsection, we show in the left panel of figure 2 the three neutrino masses $m_{1}, m_{2}$ and $m_{3}$ given in eq. (4.11), and their sum $\sum m_{i}$ as a function of the lightest neutrino mass $m_{1}$. We find that our model predicts the following ranges

$$
\begin{array}{rlrl}
0.064759 & \lesssim \sum m_{i}(\mathrm{eV}) \lesssim 0.100929, & & 0.005033 \lesssim m_{1}(\mathrm{eV}) \lesssim 0.021934 \\
0.009851 & \lesssim m_{2}(\mathrm{eV}) \lesssim 0.023530, & 0.049614 \lesssim m_{3}(\mathrm{eV}) \lesssim 0.055478
\end{array}
$$

As a result, the predicted values of $\sum m_{i}$ around the lower bound $\sim 0.064759 \mathrm{eV}$ are consistent with normal mass hierarchy which requires ${ }^{12} \sum m_{i}(\mathrm{eV}) \gtrsim 0.065431$. This lower bound of $\sum m_{i}$ may be achieved in the forthcoming experiments with further cosmological data such as CORE+BAO aiming to reach a $0.062 \mathrm{eV}$ sensitivity on the sum of the three active neutrino masses [97].

In the right panel of figure 2, we show the correlation between $m_{\beta}$ and the lightest neutrino mass $m_{1}$. The orange region is achieved by varying all the input parameters $\left(\Delta m_{i j}^{2}, a, b,|\mathrm{k}|\right.$ and $\left.\phi_{k}\right)$ in their $3 \sigma$ ranges while the red points stands for our model prediction. We find that the effective electron neutrino mass is given by

$$
0.0100158 \lesssim m_{\beta}(\mathrm{eV}) \lesssim 0.023765
$$

It is clear that our predictions for $m_{\beta}$ are too small when compared to the anticipated future $\beta$-decay experiments sensitivities such as KATRIN $(\sim 0.2 \mathrm{eV})[96]$, HOLMES $(\sim 0.1$ $\mathrm{eV})$ [98], and Project $8(\sim 0.04 \mathrm{eV})$ [99]. If the actual electron neutrino mass would be measured by one of these experiments the neutrino sector of the present model will be ruled out. Otherwise, the obtained values could be probed by new experimental projects that must aim to reach improved sensitivities around $0.01 \mathrm{eV}$.

Another possible portal to probe the scale of neutrino masses comes from experiments exploring the nature of neutrinos which is also one of the present objectives in the field of

\footnotetext{
${ }^{12}$ This bound is obtained by taking the best fit values of the mass squared differences $\Delta m_{21}^{2}$ and $\Delta m_{31}^{2}$ from ref. [3] with any value of the lightest neutrino mass $m_{1}$ obtained in eq. (5.6).
} 


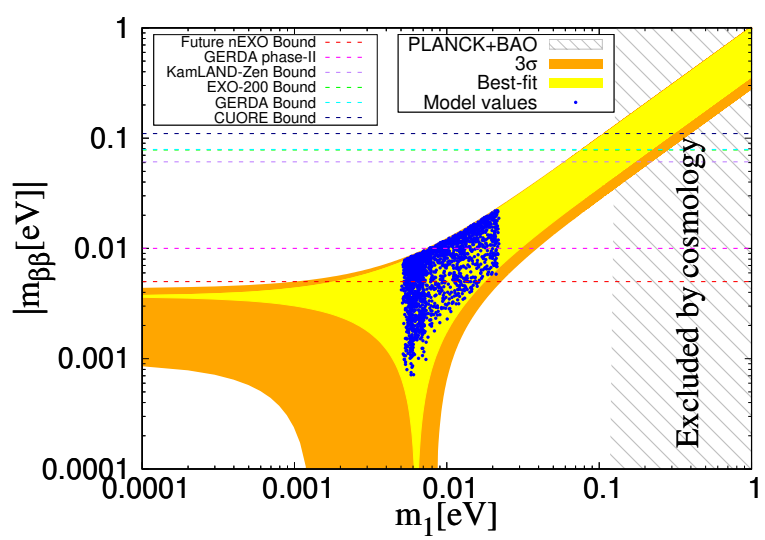

Figure 3. $\left|m_{\beta \beta}\right|$ as a function of $m_{1}$. The vertical gray dashed region indicates the upper limit on the sum of the three light neutrino masses from Planck+BAO data.

neutrino physics. Up to now, the probe of the Majorana nature of neutrinos is available only through $0 \nu \beta \beta$ decay. This is a process that violates lepton number $L$ by two units, and since there are no SM interactions that violates $L$, the discovery of $0 \nu \beta \beta$ would have interesting implications for model building beyond the SM such as the existence of a new mechanism for mass generation compared to the charged fermions obtaining their masses via the Higgs mechanism. The $0 \nu \beta \beta$ decay amplitude is proportional to the effective Majorana mass $\left|m_{\beta \beta}\right|$ defined as $\left|m_{\beta \beta}\right|=\left|\sum_{i} U_{e i}^{2} m_{i}\right|$, and may be expressed in terms of our model parameters and the parameters of the $\mathcal{U}_{\nu}$ mixing matrix

$$
\left|m_{\beta \beta}\right|=\left|\frac{2 m_{1}}{3} \cos ^{2} \theta+\frac{1}{3} \sqrt{m_{1}^{2}+\Delta m_{21}^{2}} e^{\frac{i}{2} \alpha_{21}}+\frac{2}{3} \sin ^{2} \theta \sqrt{m_{1}^{2}+\Delta m_{31}^{2}} e^{\frac{i}{2}\left(\alpha_{31}-2 \sigma\right)}\right|
$$

Notice that the relevance of the absolute mass scale in $0 \nu \beta \beta$ experiments arise from the dependence of $\left|m_{\beta \beta}\right|$ on $m_{i}$. Although $0 \nu \beta \beta$ decay has not been observed, there are dozens of running and forthcoming experiments around the world setting as their objective the detection of this process. The current limits on $\left|m_{\beta \beta}\right|$ come from the KamLANDZen [100], CUORE [101], GERDA [102] and EXO [103] experiments corresponding to $\left|m_{\beta \beta}\right|<(0.061-0.165) \mathrm{eV},\left|m_{\beta \beta}\right|<(0.075-0.35) \mathrm{eV},\left|m_{\beta \beta}\right|<(0.079-0.180) \mathrm{eV}$ and $\left|m_{\beta \beta}\right|<(0.078-0.239) \mathrm{eV}$ respectively. Figure 3 shows the correlation between $\left|m_{\beta \beta}\right|$ and the lightest neutrino mass $m_{1}$ for normal mass hierarchy. This plot is obtained by varying the oscillation parameters in their $3 \sigma$ range while the Majorana phases are varied in the range $[0 \rightarrow 2 \pi]$. The horizontal dashed lines represent the limits on $\left|m_{\beta \beta}\right|$ from current $0 \nu \beta \beta$ decay experiments while the vertical gray region is disfavored by the Planck $+\mathrm{BAO}$ data. From this figure, we extract our range of the effective Majorana mass

$$
0.000715 \lesssim\left|m_{\beta \beta}\right|(\mathrm{eV}) \lesssim 0.022028
$$

The predictions for $\left|m_{\beta \beta}\right|$ are far from the current sensitivities mentioned above, on the other hand, the next-generation experiments such as GERDA Phase II, CUPID, nEXO and SNO+-II will cover the values of $\left|m_{\beta \beta}\right|$ in eq. (5.9) as they aim for sensi- 
tivities around $\left|m_{\beta \beta}\right| \sim(0.01-0.02) \mathrm{eV}$ [104], $\left|m_{\beta \beta}\right| \sim(0.006-0.017) \mathrm{eV} \mathrm{[105],}\left|m_{\beta \beta}\right| \sim$ $(0.008-0.022) \mathrm{eV}[106]$ and $\left|m_{\beta \beta}\right| \sim(0.02-0.07) \mathrm{eV}$ [107] respectively.

\section{$6 \quad$ Leptogenesis}

In this section, we investigate the generation of the baryon asymmetry of the universe within our SUSY SU(5) $\times D_{4} \times \mathrm{U}(1)$ model in the case of normal mass hierarchy. In this scenario, the presence of three $\mathrm{RH}$ neutrinos as the key ingredients for small neutrino masses can also produce the BAU through the leptogenesis mechanism. In this case, a lepton asymmetry $Y_{L}$ (equally $B-L$ asymmetry $Y_{B-L}$ ) is generated through the out-ofequilibrium $C P$ violating decays of $\mathrm{RH}$ neutrinos $N_{i}^{c}$ (and their supersymmetric partners in SUSY models) in the early universe. This lepton asymmetry is then partially converted into the baryon asymmetry of the universe $Y_{B}$ via $(B+L)$ violating sphaleron transitions [19].

The excess of baryons over anti-baryons is evaluated through the baryon asymmetry $Y_{B}$ relative to the entropy density $s$ or the baryon asymmetry $\eta_{B}$ relative to the density of photons $n_{\gamma}$, defined respectively as

$$
Y_{B}=\frac{n_{B}-n_{\bar{B}}}{s}, \quad \eta_{B}=\frac{n_{B}-n_{\bar{B}}}{n_{\gamma}}
$$

where $n_{B}$ and $n_{\bar{B}}$ are the number densities of baryons and anti-baryons. The experimental values of these parameters obtained from the latest data from the Planck satellite are given by $Y_{B}=(8.72 \pm 0.08) \times 10^{-11}$ and $\eta_{B}=(6.13 \pm 0.04) \times 10^{-10}[73]$. In order to perform an approximate estimation of $Y_{B}$, we use the following two approaches:

- It is well-known that when the right-handed neutrino mass spectrum is hierarchical, the contribution to the lepton asymmetry can be created only by the decay of the lightest RH neutrino [13, 108, 109]. Since only the NH is allowed in our model, it is clear from eqs. (4.11) and (4.12) as well as from figure 4 that $M_{3}$ is the lightest RH neutrino where the masses of $M_{1}$ and $M_{2}$ differ at most by a factor of 3 (with $\left.3 M_{3}<M_{2}, M_{1}\right)$.

- Since all Majorana masses are above $T=10^{12}\left(1+\tan ^{2} \beta\right)$ for $\tan \beta=5$ and $\tan \beta=10$ - used in the charged fermion sector to fit the experimental data - we perform our study in the one flavor approximation where all charged leptons are out-of-equilibrium and there is no difference between them at the time leptogenesis takes place.

Taking this two points into consideration, the magnitude of $B-L$ asymmetry generated by $N_{3}$ can be parameterized as follows [110]

$$
Y_{B-L}=-\left(\varepsilon_{N_{3}} Y_{N_{3}}^{e q}+\varepsilon_{\tilde{N}_{3}} Y_{\tilde{N}_{3}}^{e q}\right) \eta_{33}
$$

where $\varepsilon_{N_{3}}\left(\varepsilon_{\tilde{N}_{3}}\right)$ is the $C P$ asymmetry produced in the decay of $N_{3}^{c}\left(\tilde{N}_{3}^{c}\right), \eta_{33}$ is the efficiency factor $^{13}$ related to the washout of the $C P$ asymmetry $\varepsilon_{N_{3}}\left(\varepsilon_{\tilde{N}_{3}}\right)$ due to $N_{3}^{c}\left(\tilde{N}_{3}^{c}\right)$ decays,

\footnotetext{
${ }^{13}$ The efficiency factor in the general formula of the $B-L$ asymmetry is written as the sum over all the lepton flavors $\sum_{\alpha, \beta} \eta_{\alpha \beta}$. In the present study where we employ the one flavor approximation, $\eta_{33}$ is the only efficiency factor relevant for leptogenesis.
} 


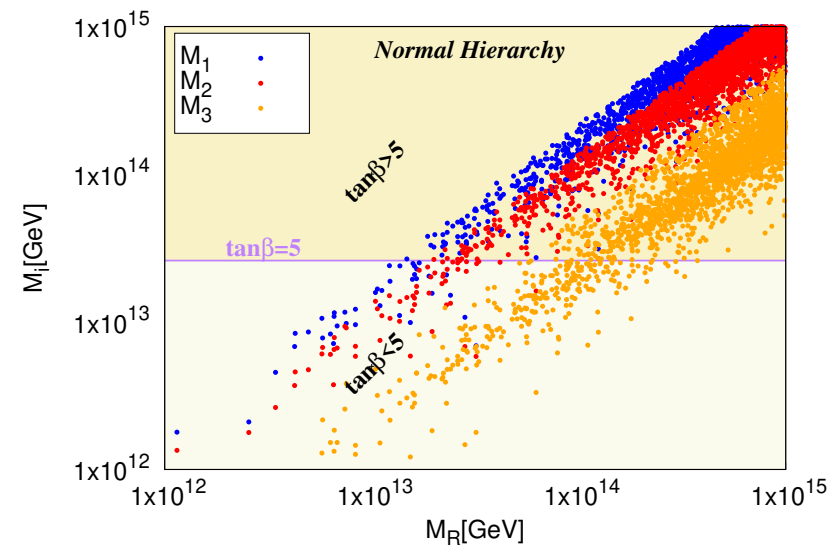

Figure 4. The RH neutrino masses $\left(M_{1}, M_{2}\right.$ and $\left.M_{3}\right)$ as a function of the Majorana mass scale $M_{R}$.

and $Y_{N_{3}}^{e q}$ is the number density of $N_{3}$ over the entropy density $\left(n_{N_{3}} / s\right)$ defined as [110]

$$
\left.Y_{N_{3}}^{e q}\right|_{T \gg M_{3}}=\frac{135 \zeta(3)}{4 \pi^{4} g_{*}}
$$

where $\zeta(3)$ denotes the Riemann zeta function and $g_{*}$ is the number of spin-degrees of freedom in thermal equilibrium; in MSSM $g_{*}=228.75$.

The first component to consider in our calculation is the source of the $C P$ asymmetry given by the $C P$ violating parameters $\varepsilon_{N_{i}}$ and $\varepsilon_{\tilde{N}_{i}}$ in $N_{i}^{c}$ and $\tilde{N}_{i}^{c}$ decays, averaged over the different decay channels $N_{i}^{c} \rightarrow L H_{u}, \tilde{L} \tilde{H}_{u}$ and $\tilde{N}_{i}^{c} \rightarrow \tilde{L} H_{u}, L \tilde{H}_{u}$ respectively. These RH neutrinos and their superpartners decay, with decay rates that reads respectively as

$$
\Gamma_{N}=\Gamma\left(N_{i}^{c} \rightarrow L H_{u}\right)+\Gamma\left(N_{i}^{c} \rightarrow \tilde{L} \tilde{H}_{u}\right) \text { and } \Gamma_{\tilde{N}}=\Gamma\left(\tilde{N}_{i}^{c} \rightarrow \tilde{L} H_{u}\right)+\Gamma\left(\tilde{N}_{i}^{c} \rightarrow L \tilde{H}_{u}\right)
$$

while the $C P$ violating parameters are given by

$$
\varepsilon_{N_{i}}=\frac{\Gamma_{N}-\bar{\Gamma}_{N}}{\Gamma_{N}+\bar{\Gamma}_{N}} \text { and } \varepsilon_{\tilde{N}_{i}}=\frac{\Gamma_{\tilde{N}}-\bar{\Gamma}_{\tilde{N}}}{\Gamma_{\tilde{N}}+\bar{\Gamma}_{\tilde{N}}}
$$

In SUSY models, the effects from the superparticles produce relatively small corrections to the BAU [111]. Therefore, by ignoring supersymmetry breaking ${ }^{14}$ —as a result of which the RH neutrinos and their superpartners have equal masses $M_{N_{i}}=M_{\tilde{N}_{i}}$, equal decay rates $\Gamma_{N_{i}}=\Gamma_{\tilde{N}_{i}}$ and equal $C P$ asymmetries $\varepsilon_{N_{i}}=\varepsilon_{\tilde{N}_{i}}[110]$-we can factorize by the $C P$ asymmetry in eq. (6.2) as $Y_{B-L}=-\varepsilon_{N_{3}}\left(Y_{N_{3}}^{e q}+Y_{\tilde{N}_{3}}^{e q}\right) \eta_{33}$. Likewise, when the equilibrium densities for leptons and sleptons are equal $Y_{N_{i}}^{e q} \approx Y_{\tilde{N}_{i}}^{e q}$, we find that the $B-L$ asymmetry parameter $Y_{B-L}$ is enhanced by a factor of 2 . Bringing together all these effects, the $C P$ asymmetry can be explicitly expressed in the one flavor approximation as

$$
\varepsilon_{N_{3}}=\frac{1}{8 \pi} \sum_{j=1,2} \frac{\operatorname{Im}\left[\left(\mathcal{Y}_{\nu} \mathcal{Y}_{\nu}^{\dagger}\right)_{j 3}^{2}\right]}{\left(\mathcal{Y}_{\nu} \mathcal{Y}_{\nu}^{\dagger}\right)_{33}} f\left(\frac{M_{j}}{M_{3}}\right)
$$

\footnotetext{
${ }^{14}$ For cases where SUSY can not be ignored see $[112,113]$.
} 
where $f(x)=\sqrt{x}(1-(1+x) \ln [(1+x) / x])$ and $\mathcal{Y}_{\nu}$ is the neutrino Yukawa coupling matrix in the basis where the Majorana mass matrix $m_{M}$ and the Yukawa matrix of the charged leptons $\mathcal{Y}_{e}$ are both diagonal. However, as explained in the appendix B, the contribution of the mixing matrix that diagonalizes $\mathcal{Y}_{e}$ leads to $C P$ asymmetry of order $\left|\varepsilon_{N_{3 i}}\right| \sim \mathcal{O}\left(10^{-12}-10^{-10}\right)$ which suppress the value of the baryon asymmetry $Y_{B}$. Therefore, in order to meet the requirements of a successful leptogenesis that produces the experimental values of $Y_{B}$, we add a correction to the leading order Dirac Yukawa matrix in eq. (4.7). To account for this correction, we introduce a new flavon field $\omega$ which transforms as $1_{+-}$under $D_{4}$ with zero $\mathrm{U}(1)$ charge, we have

$$
\delta W_{D}=\frac{\lambda_{9}}{\Lambda} N_{3,2}^{c} F_{2,3} H_{5} \omega
$$

where $\lambda_{9}$ is a complex coupling constant $\lambda_{9}=\left|\lambda_{9}\right| e^{i \phi_{\omega}}$. This effective coupling is obtained from the following renormalizable superpotential

$$
W_{D}^{r e n}=N_{3,2}^{c} F_{2,3} X_{5}+\bar{X}_{5} H_{5} \omega
$$

where $X_{5}$ is a messenger field that transforms as $\mathrm{SU}(5)$ quintet, $D_{4}$ singlet $1_{+-}$and has a $\mathrm{U}(1)$ charge equals to -8 . The contribution of $\delta W_{D}$ is small and will not provide any considerable effect in the obtained neutrino masses and mixing. When the flavon field $\omega$ acquires its VEV as $\langle\omega\rangle=v_{\omega}$, we end up with the total Yukawa mass matrix ${ }^{15}$

$$
\mathcal{Y}_{D}=Y_{D}+\delta Y_{D}=\frac{m_{D}}{v_{u}}+\delta Y_{D}=\left(\begin{array}{ccc}
\lambda_{1} & 0 & 0 \\
0 & \lambda_{1} & 0 \\
0 & 0 & \lambda_{1}
\end{array}\right)+\kappa e^{i \phi_{\omega}}\left(\begin{array}{ccc}
0 & 0 & 0 \\
0 & 0 & 1 \\
0 & 1 & 0
\end{array}\right)
$$

where $\kappa=\frac{\left|\lambda_{9}\right| v_{\omega}}{\Lambda}$ is a free parameter which should be small $(\kappa \ll 1)$ in order to produce the correct BAU. Taking into account this correction, the total Yukawa neutrino mass matrix is defined as $\mathcal{Y}_{\nu}=\mathcal{U}_{\nu}^{\dagger} \mathcal{Y}_{D}$. Thus, after calculating the product $\mathcal{Y}_{\nu} \mathcal{Y}_{\nu}^{\dagger}$ in the basis where the Majorana mass matrix is diagonal, the $C P$ asymmetry parameter $\varepsilon_{N_{3}}$ corresponding to the lightest $\mathrm{RH}$ neutrino $N_{3}$ is given approximately by

$$
\begin{aligned}
\varepsilon_{N_{3}} \simeq \frac{\kappa^{2}}{9 \pi} \cos ^{2} \phi_{\omega}[ & 2 \sin ^{2}(2 \theta) \sin ^{2}\left(\sigma-\frac{\alpha_{31}}{2}\right) f\left(\frac{\tilde{m}_{1}}{\tilde{m}_{3}}\right) \\
& \left.+\sin ^{2} \theta \sin ^{2}\left(\sigma+\frac{\left(\alpha_{21}-\alpha_{31}\right)}{2}\right) f\left(\frac{\tilde{m}_{2}}{\tilde{m}_{3}}\right)\right]
\end{aligned}
$$

where $\tilde{m}_{i}$ are the washout mass parameters expressed as $\tilde{m}_{i}=v_{u}^{2} \frac{\left(\mathcal{Y}_{\nu} \mathcal{Y}_{\nu}^{\dagger}\right)_{i i}}{M_{i}}$.

The second component to address in this computation is the efficiency factor $\eta_{33}$. A good approximation is to consider the region of $\mathrm{RH}$ neutrino masses smaller than $10^{14}$ $\mathrm{GeV}$, preventing possible washout effects from $\Delta L=2$ scattering processes. In this case,

\footnotetext{
${ }^{15}$ Notice that the total light neutrino mass matrix involving the small correction $\delta Y_{D}$ is almost similar to the one in eq. (4.1) and yields approximately to the same neutrino phenomenology.
} 

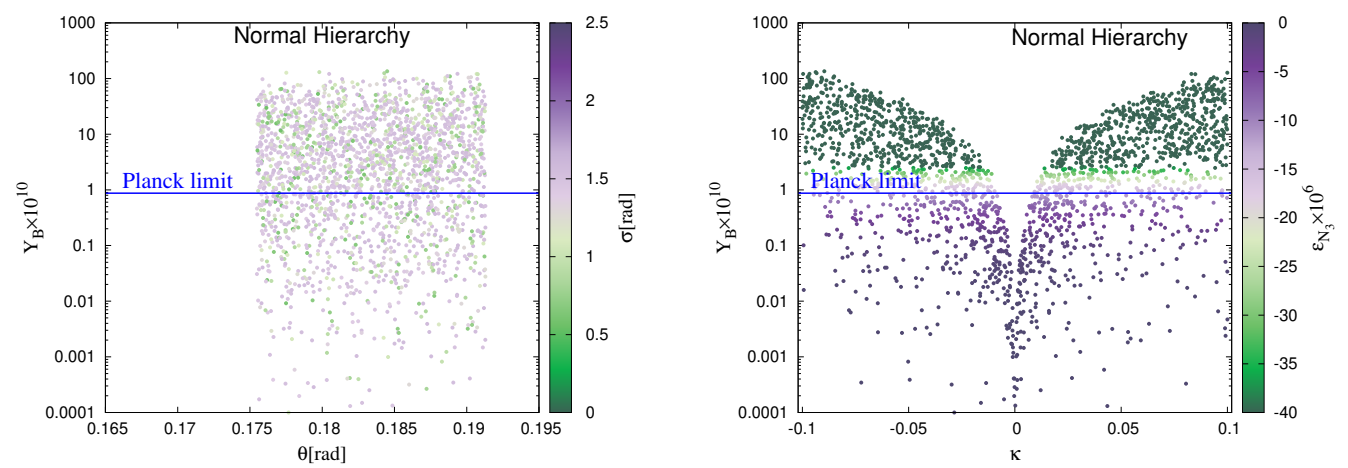

Figure 5. Left panel: $Y_{B}$ as a function of the trimaximal parameters $\theta$ and $\sigma$. Right panel: $Y_{B}$ as a function of the parameter $\kappa$ with the color code indicating the allowed range of the $C P$ asymmetry parameter $\varepsilon_{N_{3}}$. The horizontal blue line corresponds to the Planck bound on $Y_{B}$.

the efficiency factor $\eta_{33}$ can be expressed approximately as a function of the washout mass parameter $\tilde{m}_{3}$ as [110]

$$
\eta_{33} \approx\left(\frac{3.3 \times 10^{-3} \mathrm{eV}}{\tilde{m}_{3}}+\left(\frac{\tilde{m}_{3}}{0.55 \times 10^{-3} \mathrm{eV}}\right)^{1.16}\right)^{-1}
$$

Notice here that the smallness of the parameter $\kappa \ll \lambda_{1}$ implies that the washout mass parameters become approximately identical $\tilde{m}_{i} \approx m_{i}$ and hence $\tilde{m}_{3} \approx m_{3}$. Moreover, since the neutrino mass $m_{3}$ has values close to $0.5 \times 10^{-1} \mathrm{eV}$ as given in eq. (5.6), then the efficiency factor $\eta_{33}$ in our model is roughly $\eta_{33} \approx 0.5 \times 10^{-2}$.

Let us now derive the expression of the baryon asymmetry parameter $Y_{B}$. This parameter is related to lepton asymmetry $Y_{B-L}$ given in eq. (6.2) through sphaleron transitions, we have [114]

$$
Y_{B} \approx\left(\frac{8 N_{f}+4 N_{H}}{22 N_{f}+13 N_{H}}\right) Y_{B-L}=-2\left(\frac{8 N_{f}+4 N_{H}}{22 N_{f}+13 N_{H}}\right) Y_{N_{3}}^{e q} \varepsilon_{N_{3}} \eta_{33}
$$

where $N_{f}=3$ is the number of fermion generations and $N_{H}=2$ is the number of Higgs doublets in the MSSM. Accordingly, the amount of the baryon asymmetry generated in the present model is given by

$$
Y_{B} \approx-1.266 \times 10^{-3} \varepsilon_{N_{3}} \eta_{33}
$$

Therefore, $Y_{B}$ in our model depends on the trimaximal parameters $\theta$ and $\sigma$, the light neutrino masses $m_{i}$, the Majorana phases $\alpha_{31}$ and $\alpha_{21}$, as well as $\kappa$ and the phase $\phi_{\omega}$ coming from the extra contribution in the Dirac mass matrix. Using the ranges of the parameters $\theta$ and $\sigma$ restricted by the neutrino oscillation data, we show in the left panel of figure 5 the correlation between $Y_{B}, \theta$ and $\sigma$. We observe that there are many scattered points that correlate $\theta$ and $\sigma$ with the Planck bound on $Y_{B}$. On the other hand, since the parameters $\kappa$ and $\phi_{\omega}$ are not controlled by the neutrino oscillation data, we allow them to vary in the ranges $[-0.1 \rightarrow 0.1]$ and $[0 \rightarrow 2 \pi]$ respectively. Then, we plot in the right panel 

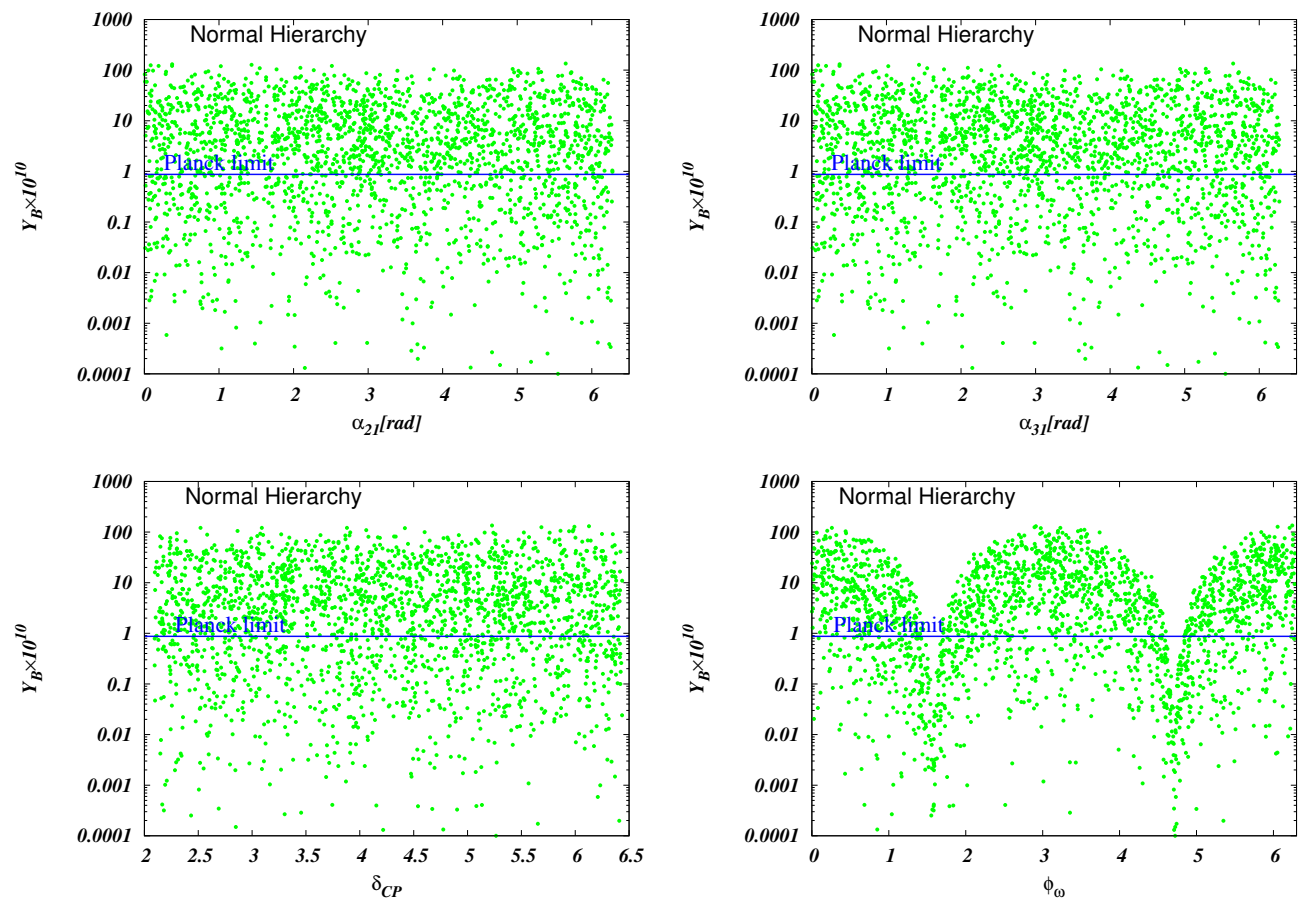

Figure 6. The baryon asymmetry $Y_{B}$ as a function of Majorana phases $\alpha_{21}$ (top left), $\alpha_{31}$ (top right), Dirac CP phase $\delta_{C P}$ (bottom left) and the high energy CP phase $\phi_{\omega}$ (bottom right). The horizontal blue line corresponds to the Planck bound.

of figure 5 the correlation between $Y_{B}$ and $\kappa$ where the color palette corresponds to the absolute value of the $C P$ asymmetry parameter $\varepsilon_{N_{3}}$. We find that the observed baryon asymmetry correspond to $\kappa$ in the range $[-0.1 \rightarrow-0.0085] \cup[0.009 \rightarrow 0.1]$ and $\left|\varepsilon_{N_{3}}\right|$ in the range $[1.302 \rightarrow 1.433] \times 10^{-5}$.

Furthermore, it is clear from the $C P$ asymmetry parameter $\varepsilon_{N_{3}}$ in eq. (6.10) that the source of $C P$ violation in the lepton sector could arise from the interplay between the low energy $C P$ phases (Dirac and Majorana phases $\delta_{C P}, \alpha_{31}$ and $\alpha_{21}$ ) and the high energy $C P$ phase $\phi_{\omega}$ originated from the complex coupling constant $\lambda_{9}$ in the Dirac mass matrix; see eq. (6.7). Therefore, we plot in figure 6 , the baryon asymmetry parameter $Y_{B}$ as a function of the low energy $C P$ phases $\left(\alpha_{31}, \alpha_{21}\right.$ and $\left.\delta_{C P}\right)$ and the high energy $C P$ phase $\phi_{\omega}$ which is the key ingredient for generating the observed range of $Y_{B}$. We observe that the ranges of the Majorana phases (top panels) and the Dirac phase (bottom left panel) are not constrained compared to their inserted intervals, nevertheless, the scattered points including the $C P$ conserving values of the Majorana phases $\alpha_{31}, \alpha_{21}=0, \pi$ - are consistent with the Planck limit on $Y_{B}$. However, even in the case of these $C P$ conserving values, $C P$ violation is guaranteed by the high energy $C P$ phase $\phi_{\omega}$. For this reason, we plot in the bottom right panel of figure 6 the correlation between $Y_{B}$ and $\phi_{\omega}$ where we find that $\phi_{\omega}$ vary within the range $0 \lesssim \phi_{\omega} \lesssim 6.279$, while the $C P$ conserving values $\phi_{\omega}=\frac{\pi}{2}$ and $\phi_{\omega}=\frac{3 \pi}{2}$ as well as the regions around them are excluded (the sections of the blue line without any 

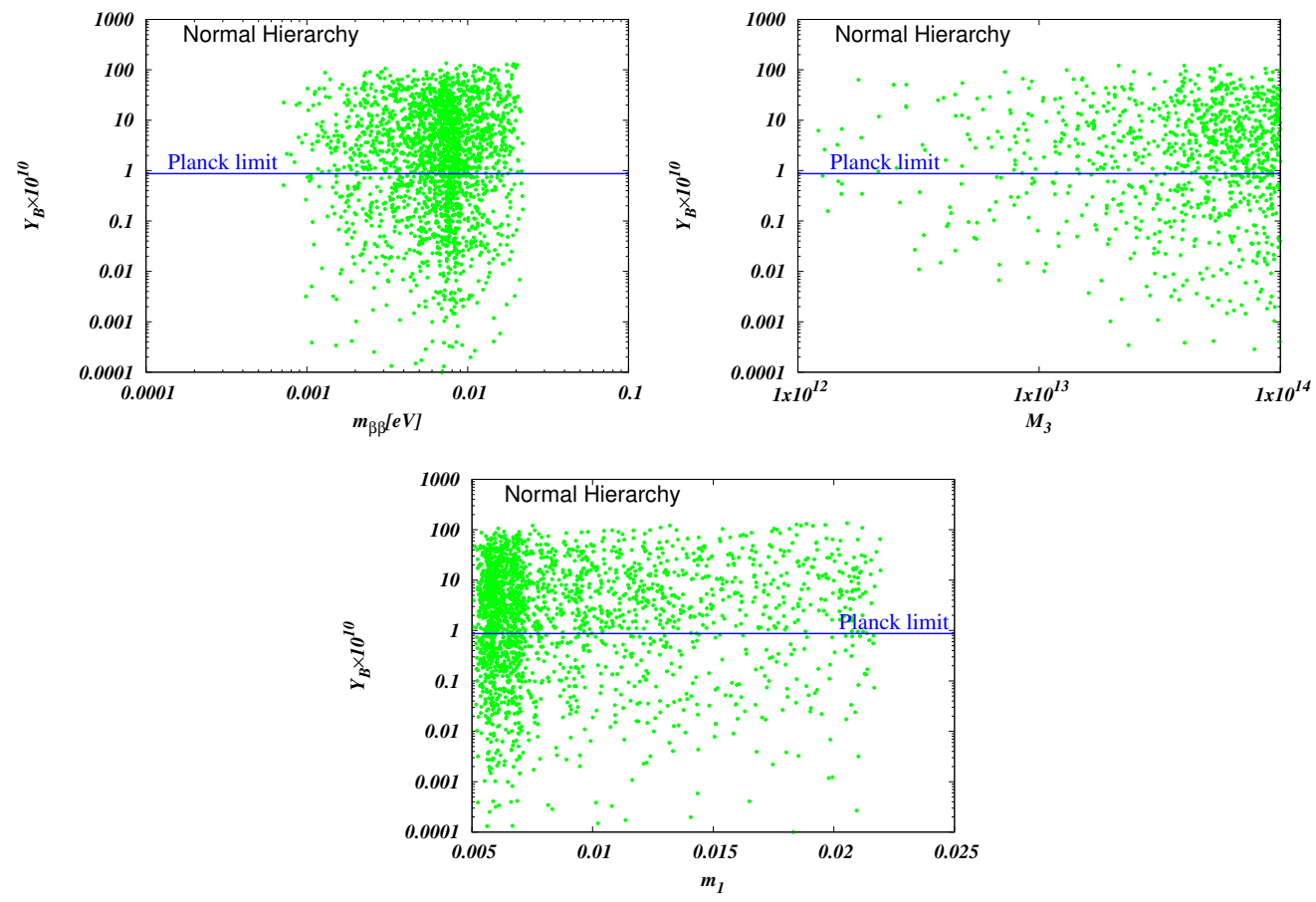

Figure 7. The baryon asymmetry $Y_{B}$ as a function of the effective Majorana mass $m_{\beta \beta}$ (top left), the lightest RH neutrino mass $M_{3}$ (top right) and the lightest neutrino mass $m_{1}$ (bottom). The horizontal blue line corresponds to the Planck bound.

points). Therefore, this source of $C P$ violation plays a crucial role in generating the baryon asymmetry in the present model.

From another point of view, since both $Y_{B}$ and $m_{\beta \beta}$ depend on the Majorana phases $\alpha_{31}$ and $\alpha_{21}$, there exists a correlation between the effective Majorana mass $m_{\beta \beta}$ which governs the $0 \nu \beta \beta$ process and the baryon asymmetry parameter $Y_{B}$. Therefore, we display in the top left of figure $7 Y_{B}$ as a function of $m_{\beta \beta}$ where we observe that there are several points satisfying the Planck limit on the baryon asymmetry parameter. Besides $m_{\beta \beta}$, the correlation of $Y_{B}$ with the lightest $\mathrm{RH}$ neutrino mass $M_{3}$ and the lightest neutrino mass $m_{1}$ is displayed, respectively, in the top right panel and the bottom panel of figure 7 , where we observe that there are several allowed points for both $M_{3}$ and $m_{1}$ within the Planck limit on $Y_{B}$.

\section{Summary and conclusion}

In this work, we have presented a model with a $D_{4}$ family symmetry to explain the fermion flavor structures in the framework of supersymmetric SU(5) grand unified theory. Besides the $\mathrm{SU}(5) \times D_{4}$ model proposed in ref. [49] — which was merely an implementation of the $D_{4}$ in SU(5) - this is the first comprehensive study of a four-dimensional SU(5) GUT with a flavor symmetry that does not include triplet irreducible representations. To establish a thorough analysis of this model, we have enlarged the field content of the usual scalar 
and matter sectors of SUSY SU(5) GUT. Explicitly, we have added three RH neutrinos to generate neutrino masses via the type I seesaw mechanism, heavy messenger fields to make the model renormalizable at the GUT scale, higher dimensional Higgs multiplets to produce realistic quark-lepton Yukawa coupling ratios, and gauge singlet flavon fields to give rise to the observed fermion mass spectrum and mixing through the spontaneous symmetry breaking of the flavor group. Moreover, after adding these fields, an additional $\mathrm{U}(1)$ symmetry is imposed to control the invariance of the superpotentials in the quark and lepton sectors, and also the dangerous $d=4$ and $d=5$ proton decay operators.

Integrating out the heavy messenger fields from the renormalizable superpotentials gives rise to higher-dimensional effective operators responsible for the fermion flavor structures. Moreover, to go beyond the minimal $\mathrm{SU}(5)$ relation $\mathcal{Y}_{e}^{T}=\mathcal{Y}_{d}$ as well as the popular GJ relations which are disfavored by the experimental results, we have considered the CG factors $y_{e} / y_{d}=4 / 9$ and $y_{\mu} / y_{s}=9 / 2$ which are realized through the coupling of messenger fields with higher 24- and 45-dimensional Higgs fields and the flavon fields. This has led to the double ratio $\frac{y_{\mu}}{y_{s}} \frac{y_{d}}{y_{e}} \simeq 10.12$ which is in good agreement with the phenomenological value at GUT scale. We have performed a numerical analysis in the down and charged lepton Yukawa sector where we have fixed our model parameters - the free parameters in the entries of the Yukawa matrices - and provided an accurate fit to the mixing angles, the Yukawa couplings and the Dirac $C P$ phase of the quark sector at the GUT scale.

The small neutrino masses are generated via the type I seesaw mechanism where the Dirac and Majorana mass matrices arise from renormalizable terms. The resulting neutrino mass matrix is of the trimaximal mixing form which is compatible with current neutrino data. By using the $3 \sigma$ experimental range of $\sin ^{2} \theta_{13}$ for both neutrino mass hierarchies we derived the range of the trimaximal mixing parameter $\theta$ where we found that only the normal mass hierarchy is allowed. Therefore, we have carried out our numerical study in this regime where we found that our model allows for $\theta_{13} \neq 0$ and $\theta_{23}<\pi / 4$ as well as excludes the conserving values of the Dirac neutrino $C P$ phase $\delta_{C P}$. We have explored the neutrino parameter space and showed numerically the predicted ranges of the nonoscillatory observables $m_{\beta}, m_{\beta \beta}$ and $\Sigma m_{i}$ that fit the $3 \sigma$ experimental range of the mixing angles and the mass squared splittings. In particular, the predicted values of $m_{\beta \beta}$ are testable at future neutrinoless double beta decay experiments.

Since the low energy $C P$ violation which manifest itself in the mixing matrix in the form of the Dirac and Majorana phases is not sufficient to describe the BAU, we have added an extra effective operator in the neutrino sector to produce the observed BAU via the leptogenesis mechanism. This operator which involves a new flavon field $\omega$ is obtained, as in the quark sector, by integrating out heavy messenger fields. Its contribution serves as a correction that perturbs the structure of the Dirac mass matrix while the high energy $C P$ phase $\phi_{\omega}$ that arises from the complex coupling constant in this operator is a new source of $C P$ violation. Therefore, we have performed a numerical study to estimate the values of the $C P$ asymmetry parameter $\varepsilon_{N_{3 i}}$ that are consistent with the baryon asymmetry parameter $Y_{B}$. We have focused on the unflavored leptogenesis approximation scenario under which the range of the lightest $\mathrm{RH}$ neutrino mass is given by $M_{3}(\mathrm{GeV}) \in\left[2.6 \times 10^{13} \rightarrow 10^{14}\right]$. We found that the $C P$ asymmetry parameter $\varepsilon_{N_{3}}$ is mainly related to the high energy 


\begin{tabular}{l|l|l|l|l|l|l|l}
\hline Messenger fields & $X_{1}$ & $X_{2}$ & $X_{3}$ & $X_{4}$ & $X_{5}$ & $Y_{1}$ & $Y_{2}$ \\
\hline$S U(5)$ & 5 & 5 & 5 & 5 & 5 & 10 & 10 \\
\hline$D_{4}$ & $1_{+,-}$ & $1_{+,+}$ & $\left(\begin{array}{c}0 \\
X_{3}\end{array}\right)$ & $1_{+,-}$ & $1_{+,-}$ & $1_{+,-}$ & $1_{+,-}$ \\
\hline$U(1)$ & -4 & 10 & -13 & 8 & -8 & 2 & -4 \\
\hline
\end{tabular}

Table 8. Messenger fields relevant in our model and their $D_{4}$ representations as well as their $\mathrm{U}(1)$ charges. The messenger fields $X_{i}$ live in 5-dimensional representation while $Y_{i}$ live in 10-dimensional representation. We assume that their mass is around the GUT scale.

$C P$ phase $\phi_{\omega}$. Therefore, we showed through scatter plots that the $C P$ conserving values $\phi_{\omega}=\frac{\pi}{2}$ and $\phi_{\omega}=\frac{3 \pi}{2}$ as well as the regions around them are excluded, while the lepton asymmetry parameter $\varepsilon_{N_{3 i}}$ must be of order $\left|\varepsilon_{N_{3 i}}\right| \sim O\left(10^{-5}\right)$ to satisfy the Planck limit on $Y_{B}$.

\section{A Messenger sector}

In this appendix we discuss the renormalizable superpotentials of all the fermions including their Feynman diagrams to obtain the higher dimensional operators relevant for the Yukawa mass matrices. The complete list of the messenger field content including their $\mathrm{SU}(5)$ and $D_{4}$ representations as well as their $U(1)$ charges is given in table 8 . To be precise, the messenger fields $Y_{i}$ are relevant for the up quark sector while $X_{i}$ are involved in the down quark, the charged lepton and the Dirac neutrino sectors. The renormalizable superpotential invariant under $D_{4} \times \mathrm{U}(1)$ associated to the up quarks reads as

$$
\begin{aligned}
W_{u p}^{R e n}= & H_{5} T_{1} Y_{1}+\bar{Y}_{1} T_{1} \xi_{1}+H_{5} T_{1} Y_{1}+\bar{Y}_{1} T_{2} \xi_{2}+H_{5} T_{1} Y_{1} \\
& +\bar{Y}_{1} T_{3} \xi_{3}+H_{5} T_{2} Y_{2}+\bar{Y}_{2} T_{2} \xi_{4}+H_{5} T_{2} Y_{2}+\bar{Y}_{2} T_{3} \xi_{5}
\end{aligned}
$$

where we have omitted the coupling constants from all terms for simplicity. The couplings in this superpotential are illustrated by the Feynman diagrams provided in figure 8. After integrating out the messenger fields $Y_{i}$ and $\bar{Y}_{i}$ from $W_{u p}^{\text {Ren }}$ we obtain the effective superpotential responsible for the masses of the up quarks given in eq. (3.1). As for the down-type quark and charged lepton sector, the renormalizable superpotential involving the five-plets messenger fields $X_{i}$ and $\bar{X}_{i}$ is given by

$$
\begin{aligned}
W_{d, e}^{R e n}= & F_{1} \phi X_{1}+X_{1} H_{24} \bar{X}_{1}+\bar{X}_{1} \varphi X_{2}+X_{2} H_{24} \bar{X}_{2}+\bar{X}_{2} H_{\overline{5}} T_{1}+F_{2,3} \Phi X_{1} \\
& +X_{1} H_{24} \bar{X}_{1}+\bar{X}_{1} \varphi X_{2}+X_{2} H_{24} \bar{X}_{2}+\bar{X}_{2} H_{\overline{5}} T_{1}+F_{2,3} H_{24} X_{3} \\
& +\bar{X}_{3} \Phi X_{1}+\bar{X}_{1} H_{\overline{45}} T_{2}+F_{2,3} \Omega X_{4}+\bar{X}_{4} H_{\overline{5}} T_{3}
\end{aligned}
$$

while the renormalizable terms relevant for the effective operator responsible for generating a successful BAU is given as follows

$$
W_{D}^{r e n}=N_{3,2}^{c} F_{2,3} X_{5}+\bar{X}_{5} H_{5} \omega
$$




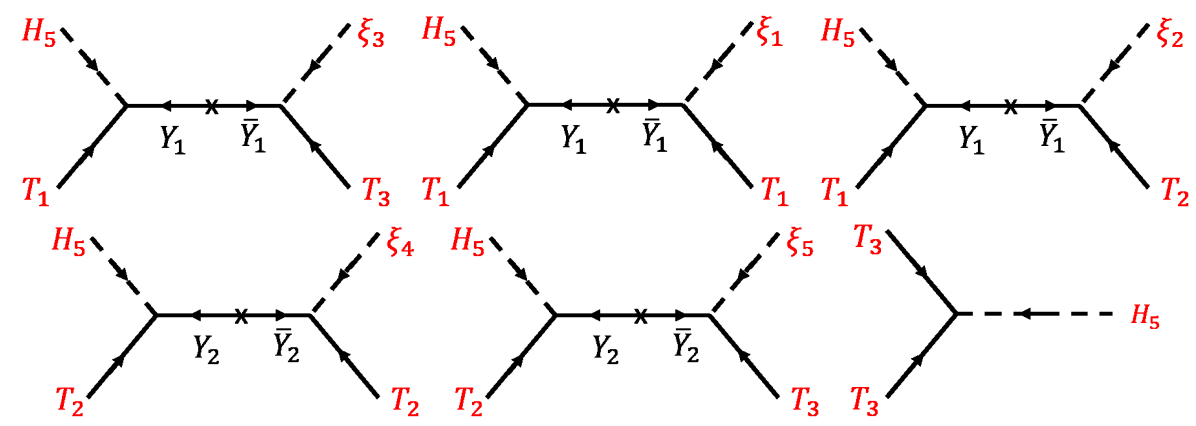

Figure 8. Diagrams inducing effective operators for the up-type quark sector. The last diagram implies that the top quark mass originate from the renormalizable operator $T_{3} T_{3} H_{5}$.

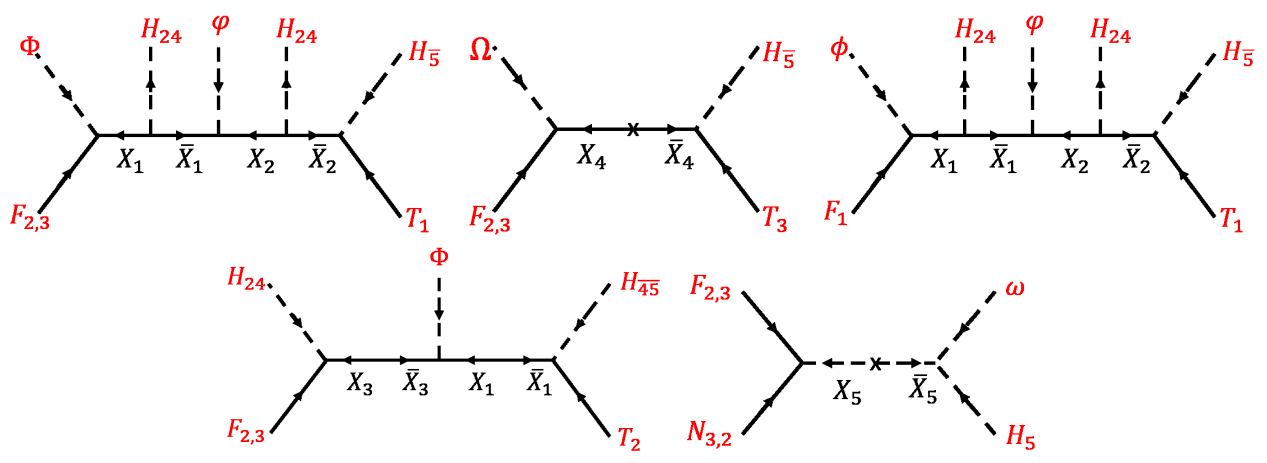

Figure 9. Diagrams inducing effective operators for the down-type quark and lepton sector. The last diagram is associated to the small Dirac correction.

Once more, integrating out the these heavy messenger fields give rise to the effective superpotentials of the down quarks, the charged leptons and the Dirac neutrino; see eqs. (3.3) and (6.7). The mass terms of the messenger fields takes the form $W_{M F}=$ $M_{X_{i}} X_{i} \bar{X}_{i}+M_{Y_{j}} Y_{j} \bar{Y}_{j}$ where $i=1,2, . .5$ and $j=1,2$. In fact, $\bar{X}_{i}$ and $\bar{Y}_{j}$ are the corresponding fields of $X_{i}$ and $Y_{j}$, they are hosted by the SU(5) representations $\overline{5}$ and 10 respectively, and they have the same $D_{4}$ representations as their partners but with opposite U(1) charges. The Feynman diagrams relevant for the superpotetials (A.2) and (A.3) are illustrated in figure 9 .

\section{B $\quad C P$ asymmetry from the charged lepton mixing and $d=6$ Dirac operators}

In this appendix, we show that the contribution of the charged lepton mixing matrix to the Yukawa mass matrix in eq. (4.7), before adding the correction $\delta Y_{D}$, cannot accommodate the observed value of the BAU. Starting with the $C P$ asymmetry formula in eq. (6.6) which can be explicitly expressed in the one flavor approximation as

$$
\varepsilon_{N_{3}^{c}}=\frac{1}{8 \pi}\left\{\frac{\operatorname{Im}\left[\left(\mathcal{Y}_{\nu} \mathcal{Y}_{\nu}^{\dagger}\right)_{13}^{2}\right]}{\left(\mathcal{Y}_{\nu} \mathcal{Y}_{\nu}^{\dagger}\right)_{33}} f\left(\frac{M_{1}}{M_{3}}\right)+\frac{\operatorname{Im}\left[\left(\mathcal{Y}_{\nu} \mathcal{Y}_{\nu}^{\dagger}\right)_{23}^{2}\right]}{\left(\mathcal{Y}_{\nu} \mathcal{Y}_{\nu}^{\dagger}\right)_{33}} f\left(\frac{M_{2}}{M_{3}}\right)\right\}
$$


where $\mathcal{Y}_{\nu}=\mathcal{U}_{T M_{2}}^{\dagger} Y_{D} \mathcal{U}_{l}$ is the neutrino Yukawa coupling matrix in the basis where the Majorana mass matrix $m_{M}$ and the Yukawa matrix of the charged leptons $\mathcal{Y}_{e}$ are both diagonal - see section $4-$ with $^{16}$

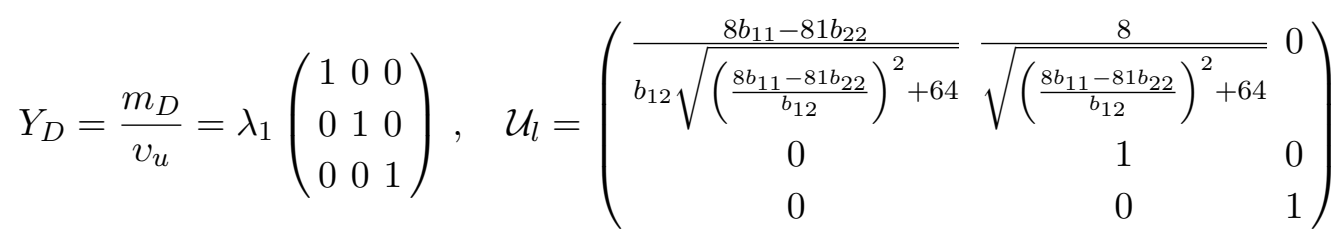

For our calculations, the values of the free parameters $b_{i j}$ are fixed by their values in the case of $\tan \beta=5$ as reported in table 6 . As a result, by inserting the expression of $\mathcal{Y}_{\nu}$ in eq. (B.1), we find that the $C P$ asymmetry parameter $\varepsilon_{N_{3}^{c}}$ depends on the coupling constant $\lambda_{1}$, the trimaximal mixing parameters $\theta$ and $\sigma$, the Majorana $C P$ phases $\alpha_{31}$ and $\alpha_{21}$ as well as the light neutrino masses $m_{i=1,2,3}$. Approximately, $\varepsilon_{N_{3}^{c}}$ is given as

$$
\begin{aligned}
\varepsilon_{N_{3}^{c}}= & 10^{-6} \frac{\lambda_{1}^{2}}{8 \pi}\left\{\left[6.194 \sin ^{4} \theta \sin ^{2}\left(\frac{\alpha_{31}}{2}\right) \cos ^{2} \sigma \sin ^{2} \sigma\right] f\left(\frac{m_{1}}{m_{3}}\right)\right. \\
& \left.+\left[2.3226 \sin ^{2} \theta\left(\cos \sigma \cos \left(\frac{\alpha_{21}-\alpha_{31}}{2}\right)+\sin \sigma \sin \left(\frac{\alpha_{21}-\alpha_{31}}{2}\right)\right)^{2}\right] f\left(\frac{m_{2}}{m_{3}}\right)\right\}
\end{aligned}
$$

Assuming that the coupling constant $\lambda_{1}$ is of order one and taking into account the obtained regions of the parameters $\theta$ and $\sigma$, the Majorana $C P$ phases $\alpha_{31}$ and $\alpha_{21}$ as well as the neutrino masses $m_{i=1,2,3}$, the baryon asymmetry parameter $\left|\varepsilon_{N_{3}^{c}}\right|$ is up to order $\mathcal{O}\left(10^{-12}-\right.$ $\left.10^{-10}\right)$. However, as discussed in section 6 , to generate the observed baryon asymmetry, the parameter $\left|\varepsilon_{N_{3}^{c}}\right|$ must be of order $\mathcal{O}\left(10^{-5}\right)$. Therefore, the charged lepton contribution to the $C P$ asymmetry parameter $\left|\varepsilon_{N_{3}^{c}}\right|$ is too small and subsequently the baryon asymmetry parameter $Y_{B}$ is strongly suppressed.

Before we close this appendix, we discuss the possibility of producing a successful leptogenesis using higher dimensional Yukawa operators as an alternative to the additional coupling in eq. (6.7). Using the charge assignments of $D_{4}$ and $\mathrm{U}(1)$ symmetries, we find that there are three invariant six dimensional operators that can be used as a correction to the leading order Dirac Yukawa matrix

$$
\frac{1}{\Lambda^{2}} N_{3,2}^{c} F_{2,3} H_{5} \rho_{i} \xi_{2} \quad \text { where } \quad i=1,2,3
$$

Since our model is renormalizable, these operators must be derived from renormalizable Yukawa couplings involving the existing messenger fields listed in table 8. For example, generating the operator $\frac{1}{\Lambda^{2}} N_{3,2}^{c} F_{2,3} H_{5} \rho_{2} \xi_{2}$ calls for a new messenger field $X_{6}$ which transforms as $\mathrm{SU}(5)$ quintet, $D_{4}$ singlet $1_{++}$and has a $\mathrm{U}(1)$ charge equals to -18 . Nevertheless, the absence of this messenger field in our model forbids the existence of this operator.

On the other hand, even if we add messenger fields to allow the operators in eq. (B.3), we end up with a highly suppressed contribution to the $C P$ asymmetry parameter $\left|\varepsilon_{N_{3}^{c}}\right|$. As

\footnotetext{
${ }^{16}$ The diagonalization of $\mathcal{Y}_{e}$ given in eq. (3.7) is obtained as a function of $b_{11}, b_{12}$ and $b_{22}$. Then, we replace these parameters by their numerical values given in table 4 to produce $\mathcal{U}_{l}$.
} 
a verification, we use the same example as above where the renormalizable superpotential which induces the effective coupling $\frac{\lambda_{10}}{\Lambda^{2}} N_{3,2}^{c} F_{2,3} H_{5} \rho_{2} \xi_{2}$ is given by

$$
W_{D}^{r e n}=N_{3,2}^{c} F_{2,3} X_{5}+\bar{X}_{5} \rho_{2} X_{6}+\bar{X}_{6} H_{5} \xi_{2},
$$

where the coupling constants are omitted for simplicity. Subsequently, the total Yukawa mass matrix reads as

$$
\mathcal{Y}_{D}=Y_{D}+\delta Y_{D}=\frac{m_{D}}{v_{u}}+\delta Y_{D}=\left(\begin{array}{ccc}
\lambda_{1} & 0 & 0 \\
0 & \lambda_{1} & 0 \\
0 & 0 & \lambda_{1}
\end{array}\right)+\frac{\left|\lambda_{10}\right| v_{\rho_{2}} v_{\xi_{2}}}{\Lambda^{2}}\left(\begin{array}{lll}
0 & 0 & 0 \\
0 & 0 & 1 \\
0 & 1 & 0
\end{array}\right) e^{i \phi_{H}}
$$

where $\lambda_{10}$ is a complex coupling constant $\lambda_{10}=\left|\lambda_{10}\right| e^{i \phi_{H}}$. The $C P$ asymmetry parameter $\varepsilon_{N_{3}}$ corresponding to the lightest $\mathrm{RH}$ neutrino $N_{3}$ is given approximately by

$$
\begin{aligned}
\varepsilon_{N_{3}} \simeq & \frac{1}{9 \pi}\left(\frac{\left|\lambda_{10}\right| v_{\rho_{2}} v_{\xi_{2}}}{\Lambda^{2}}\right)^{2} \cos ^{2} \phi_{H}\left[2 \sin ^{2}(2 \theta) \sin ^{2}\left(\sigma-\frac{\alpha_{31}}{2}\right) f\left(\frac{m_{1}}{m_{3}}\right)\right. \\
& \left.+\sin ^{2} \theta \sin ^{2}\left(\sigma+\frac{\left(\alpha_{21}-\alpha_{31}\right)}{2}\right) f\left(\frac{m_{2}}{m_{3}}\right)\right]
\end{aligned}
$$

The obtained $C P$ asymmetry parameter $\varepsilon_{N_{3}}$ is proportional to the factor $\varepsilon_{N_{3}} \sim\left(\frac{\left|\lambda_{10}\right| v_{\rho_{2}} v_{\xi_{2}}}{\Lambda^{2}}\right)^{2}$ which involves the flavon VEV $\frac{v_{\rho_{2}}}{\Lambda}$ from the neutrino sector as well as $\frac{v_{\xi_{2}}}{\Lambda}$ from the up-quark sector. According to the numerical analysis we have performed in the two sectors, we derive the interval of the ratio $\frac{\lambda_{7} v_{\rho_{2}}}{\Lambda}=-\frac{\mathrm{k}}{2} \simeq[-0.27557,0.26298]$ while the size of the flavon VEV $\frac{\lambda_{12}^{u} v_{\xi_{2}}}{\Lambda}=a_{12} \simeq 0.1530 \times 10^{-2}$ in the case of $\tan \beta=10 .{ }^{17}$ In order to get an estimate on the obtained $C P$ asymmetry parameter $\varepsilon_{N_{3}}$ in eq. (B.6), we assume, as is reasonable to do, that the coupling constants $\lambda_{7}, \lambda_{12}^{u}$ and $\left|\lambda_{10}\right|$ are of order one and we allow the phase $\phi_{H}$ to vary in the interval $[0,2 \pi]$. Therefore, we find that the $C P$ asymmetry parameter $\left|\varepsilon_{N_{3}^{c}}\right|$ is up to order $\mathcal{O}\left(10^{-12}-10^{-7}\right)$ which is too small to account for a successful leptogenesis. As a result, the baryon asymmetry parameter $Y_{B}$ is strongly suppressed when addressing leptogenesis through the six dimensional operator $\frac{\lambda_{10}}{\Lambda^{2}} N_{3,2}^{c} F_{2,3} H_{5} \rho_{2} \xi_{2}$. The same discussion holds for the other two operators $\frac{1}{\Lambda^{2}} N_{3,2}^{c} F_{2,3} H_{5} \rho_{1} \xi_{2}$ and $\frac{1}{\Lambda^{2}} N_{3,2}^{c} F_{2,3} H_{5} \rho_{3} \xi_{2}$.

\section{Some aspects of the dihedral group $D_{4}$}

The dihedral group $D_{4}$ is a finite group that is generated by the reflection $t$ and the $45^{\circ}$ rotation $s$ satisfying $s^{4}=t^{2}=I$ and $t s t=s^{-1}$. A rotation followed by a reflection is different than a reflection followed by a rotation which means that the two generators $s$ and $t$ do not commute with each other. This non-Abelian group has 5 irreducible representations $R_{i=1, \ldots, 5}$ : one doublet denoted as $2_{0,0}$, and four singlets denoted as $1_{+,+}$(the trivial singlet), $1_{+,-} 1_{-,+}$and $1_{-,-}$. The indices of these representations represent their characters under

\footnotetext{
${ }^{17}$ Notice that the value of the flavon VEV $\frac{\lambda_{12}^{u} v_{\xi_{2}}}{\Lambda}=a_{12} \simeq 0.15866 \times 10^{-3}$ in the case of $\tan \beta=5$ is much smaller and therefore the estimate on the $C P$ asymmetry parameter $\varepsilon_{N_{3}}$ becomes much suppressed.
} 
the two generators $t$ and $s$ as in the following table

\begin{tabular}{llllll}
\hline$\chi_{R_{i}}$ & $\chi_{2_{0,0}}$ & $\chi_{1_{+,+}}$ & $\chi_{1_{+,-}}$ & $\chi_{1_{-,+}}$ & $\chi_{1_{-,-}}$ \\
\hline \hline$t$ & 0 & +1 & +1 & -1 & -1 \\
\hline$s$ & 0 & +1 & -1 & +1 & -1 \\
\hline
\end{tabular}

The squares of the dimensions of these irreducible representations are related to the order 8 of the $D_{4}$ group through the formula $8=1_{+,+}^{2}+1_{+,-}^{2}+1_{-,+}^{2}+1_{-,-}^{2}+2_{0,0}$. Let us now turn to the tensor products among the irreducible representations of $D_{4}$. The tensor product between two $D_{4}$ doublets is decomposed into a sum of the four singlet representations of $D_{4}$ as

$$
\begin{aligned}
\left(\begin{array}{l}
x_{1} \\
x_{2}
\end{array}\right)_{2_{0,0}} \otimes\left(\begin{array}{l}
y_{1} \\
y_{2}
\end{array}\right)_{2_{0,0}}= & \left(x_{1} y_{2}+x_{2} y_{1}\right)_{1_{+,+}} \oplus\left(x_{1} y_{1}+x_{2} y_{2}\right)_{1_{+,-}} \oplus\left(x_{1} y_{2}-x_{2} y_{1}\right)_{1_{-,+}} \\
& \oplus\left(x_{1} y_{1}-x_{2} y_{2}\right)_{1_{-,-}}
\end{aligned}
$$

while the tensor products among the singlet representations can be expressed as

$$
1_{d, e} \otimes 1_{f, g}=1_{d f, e g} \quad \text { with } \quad d, e, f, g= \pm
$$

For more details on the $D_{4}$ group, see, e.g., [36].

\section{Vacuum alignment of $D_{4}$ flavon doublets}

Establishing an origin of the VEV directions is an essential part when using non-Abelian discrete flavor symmetries to build models of fermion masses and mixing. In our model, the VEVs of the $D_{4}$ doublet flavons pointing in the directions given in eqs. (3.5) and (4.3) were assumed in order to produce the charged fermions and neutrino masses consistent with the experimental data. One of the well-known approaches to check if these VEV directions are a solution of the scalar potential is by introducing a set of alignment fields called driving fields and a continuous $\mathrm{U}(1)_{R}$ symmetry. Under such a symmetry, the matter superfields including right-handed neutrinos carry charge +1 , flavons and Higgs fields are uncharged while the driving fields have charge +2 [115]. As a result of the these $\mathrm{U}(1)_{R}$ charge assignments, the driving fields couple only to flavons and appear linearly in the superpotential, while the vacuum alignment is obtained by setting their F-terms to zero. In general, the alignment through F-terms provide also relations between flavons VEVs. Here, we introduce two driving fields denoted as $\chi_{q}$ and $\chi_{\nu}$ transforming under $D_{4} \times \mathrm{U}(1)$ as

$$
\chi_{q} \sim\left(\left\{1_{-,-}\right\},\{30\}\right), \quad \chi_{\nu} \sim\left(\left\{1_{-,+}\right\},\{-20\}\right)
$$

The renormalizable terms involving these driving fields invariant under the flavor symmetry $D_{4} \times \mathrm{U}(1)$ are given by

$$
\mathcal{W}_{d}=c_{1} \chi_{q}(\Omega \Phi)_{1_{-,-}}+c_{2} \chi_{\nu}(\Gamma \digamma)_{1_{-,+}}+c_{3} \chi_{\nu}(\digamma \digamma)_{1_{-,+}}+c_{4} \chi_{\nu}(\Gamma \Gamma)_{1_{-,+}}+c_{5} \chi_{\nu} \rho_{2} \rho_{3}
$$


In the SUSY limit where the F-terms of $\chi_{q}$ and $\chi_{\nu}$ vanish, the condition for the minima are

$$
\begin{aligned}
& \frac{\partial \mathcal{W}_{d}}{\partial \chi_{q}}=c_{1}\left(\Omega_{1} \Phi_{1}-\Omega_{2} \Phi_{2}\right)=0 \\
& \frac{\partial \mathcal{W}_{d}}{\partial \chi_{\nu}}=c_{2}\left(\Gamma_{1} \digamma_{2}-\Gamma_{2} \digamma_{1}\right)+c_{3}\left(\digamma_{1} \digamma_{2}-\digamma_{2} \digamma_{1}\right)+c_{4}\left(\Gamma_{1} \Gamma_{2}-\Gamma_{2} \Gamma_{1}\right)+c_{5} \rho_{2} \rho_{3}=0
\end{aligned}
$$

Clearly, the first equation admits three different solutions given by

$$
\begin{aligned}
& \text { (1) : } \quad\langle\Phi\rangle=\left(v_{\Phi}, v_{\Phi}\right)^{T}, \quad\langle\Omega\rangle=\left(v_{\Omega}, v_{\Omega}\right)^{T} \\
& \text { (2): } \quad\langle\Phi\rangle=\left(0, v_{\Phi}\right)^{T}, \quad\langle\Omega\rangle=\left(v_{\Omega}, 0\right)^{T} \\
& \text { (3): } \quad\langle\Phi\rangle=\left(v_{\Phi}, 0\right)^{T}, \quad\langle\Omega\rangle=\left(0, v_{\Omega}\right)^{T}
\end{aligned}
$$

where the last solution is the one we have chosen to generate the Yukawa matrices of the down-type quarks $\mathcal{Y}_{d}$ and charged leptons $\mathcal{Y}_{e}$ in eqs. (3.6) and (3.7), respectively. As for the second equation in (D.3), it admits the VEV direction given by

$$
\left\langle\rho_{2}\right\rangle=v_{\rho_{2}}, \quad\left\langle\rho_{3}\right\rangle=v_{\rho_{3}}, \quad\langle\digamma\rangle=\left(v_{\digamma}, v_{\digamma}\right)^{T}, \quad\langle\Gamma\rangle=\left(0, v_{\Gamma}\right)^{T},
$$

which we have used to produce the Majorana mass matrix provided the following relation between the involved VEVs holds

$$
v_{\digamma}=\frac{c_{5}}{c_{2}} \frac{v_{\rho_{2}} v_{\rho_{3}}}{v_{\Gamma}}
$$

According to the assumptions we have adopted to obtain the total neutrino mass matrix see eqs. (4.5) and (4.6) - it follows that the set of flavon VEVs $\left\{v_{\rho_{1}}, v_{\digamma}\right\}$ and $\left\{v_{\rho_{2}}, v_{\rho_{3}}, v_{\Gamma}\right\}$ are of the same order of magnitude. Moreover, the flavon VEVs $v_{\rho_{2}}$ and $v_{\digamma}$ are related in eq. (D.5) through the couplings $c_{2}$ and $c_{5}$ which we assume that they are of the same order. As a result, we deduce that all the flavons used in the neutrino sector are comparable to each other which is in agreement with the numerical analysis performed in section 5 .

On the contrary, the first equation in (D.3) responsible for aligning the flavon doublets $\Omega$ and $\Phi$ does not induce any relation between their VEVs $v_{\Omega}$ and $v_{\Phi}$. This is clearly reasonable since they are not of the same order of magnitude as discussed numerically in section 5. These two flavon VEVs contribute respectively to the second and the third generations of down quarks (charged leptons) which are strongly hierarchical.

Open Access. This article is distributed under the terms of the Creative Commons Attribution License (CC-BY 4.0), which permits any use, distribution and reproduction in any medium, provided the original author(s) and source are credited.

\section{References}

[1] T. Kajita, Nobel Lecture: Discovery of atmospheric neutrino oscillations, Rev. Mod. Phys. 88 (2016) 030501.

[2] A.B. McDonald, Nobel Lecture: The Sudbury Neutrino Observatory: Observation of flavor change for solar neutrinos, Rev. Mod. Phys. 88 (2016) 030502. 
[3] I. Esteban, M.C. Gonzalez-Garcia, M. Maltoni, T. Schwetz and A. Zhou, The fate of hints: updated global analysis of three-flavor neutrino oscillations, JHEP 09 (2020) 178 [arXiv:2007.14792] [INSPIRE].

[4] F. Capozzi, E. Di Valentino, E. Lisi, A. Marrone, A. Melchiorri and A. Palazzo, Global constraints on absolute neutrino masses and their ordering, Phys. Rev. D 95 (2017) 096014 [Addendum ibid. 101 (2020) 116013] [arXiv:2003.08511] [INSPIRE].

[5] P.F. de Salas et al., 2020 global reassessment of the neutrino oscillation picture, JHEP 02 (2021) 071 [arXiv:2006.11237] [INSPIRE].

[6] T2K collaboration, Constraint on the matter-antimatter symmetry-violating phase in neutrino oscillations, Nature 580 (2020) 339 [Erratum ibid. 583 (2020) E16] [arXiv: 1910.03887] [INSPIRE].

[7] A.D. Sakharov, Violation of CP Invariance, C asymmetry, and baryon asymmetry of the universe, Pisma Zh. Eksp. Teor. Fiz. 5 (1967) 32 [JETP Lett. 5 (1967) 24] [Sov. Phys. Usp. 34 (1991) 392] [Usp. Fiz. Nauk 161 (1991) 61] [INSPIRE].

[8] M. Dine and A. Kusenko, The origin of the matter-antimatter asymmetry, Rev. Mod. Phys. 76 (2003) 1 [hep-ph/0303065] [InSPIRE].

[9] W. Buchmüller, R.D. Peccei and T. Yanagida, Leptogenesis as the origin of matter, Ann. Rev. Nucl. Part. Sci. 55 (2005) 311 [hep-ph/0502169] [INSPIRE].

[10] W. Buchmüller, Baryogenesis, Dark Matter and the Maximal Temperature of the Early Universe, Acta Phys. Polon. B 43 (2012) [arXiv:1212.3554] [InSPIRE].

[11] A.D. Dolgov, NonGUT baryogenesis, Phys. Rept. 222 (1992) 309 [INSPIRE].

[12] A.D. Dolgov and Y.B. Zeldovich, Cosmology and Elementary Particles, Rev. Mod. Phys. 53 (1981) 1 [INSPIRE].

[13] M. Fukugita and T. Yanagida, Baryogenesis Without Grand Unification, Phys. Lett. B 174 (1986) 45 [INSPIRE].

[14] P. Minkowski, $\mu \rightarrow e \gamma$ at a Rate of One Out of $10^{9}$ Muon Decays?, Phys. Lett. B 67 (1977) 421 [INSPIRE].

[15] T. Yanagida, Horizontal gauge symmetry and masses of neutrinos, Conf. Proc. C 7902131 (1979) 95 [INSPIRE].

[16] T. Yanagida, Horizontal Symmetry and Masses of Neutrinos, Prog. Theor. Phys. 64 (1980) 1103 [INSPIRE].

[17] M. Gell-Mann, P. Ramond and R. Slansky, Complex Spinors and Unified Theories, Conf. Proc. C 790927 (1979) 315 [arXiv:1306.4669] [INSPIRE].

[18] R.N. Mohapatra and G. Senjanović, Neutrino Mass and Spontaneous Parity Nonconservation, Phys. Rev. Lett. 44 (1980) 912 [INSPIRE].

[19] S.Y. Khlebnikov and M.E. Shaposhnikov, The Statistical Theory of Anomalous Fermion Number Nonconservation, Nucl. Phys. B 308 (1988) 885 [INSPIRE].

[20] J.C. Pati and A. Salam, Lepton Number as the Fourth Color, Phys. Rev. D 10 (1974) 275 [Erratum ibid. 11 (1975) 703] [INSPIRE].

[21] H. Georgi and S.L. Glashow, Unity of All Elementary Particle Forces, Phys. Rev. Lett. 32 (1974) 438 [INSPIRE]. 
[22] H. Georgi, H.R. Quinn and S. Weinberg, Hierarchy of Interactions in Unified Gauge Theories, Phys. Rev. Lett. 33 (1974) 451 [inSPIRE].

[23] H. Georgi, The State of the Art - Gauge Theories, AIP Conf. Proc. 23 (1975) 575 [INSPIRE].

[24] H. Fritzsch and P. Minkowski, Unified Interactions of Leptons and Hadrons, Annals Phys. 93 (1975) 193 [INSPIRE].

[25] S. Raby, Supersymmetric Grand Unified Theories: From Quarks to Strings via SUSY GUTs, vol. 939, Springer (2017), [DOI] [INSPIRE].

[26] J.R. Ellis, D.V. Nanopoulos and S. Rudaz, GUTs 3: SUSY GUTs 2, Nucl. Phys. B 202 (1982) 43 [INSPIRE].

[27] P. Langacker, Precision tests of the standard model, in Boston 1990, Proceedings, Particles, strings and cosmology, pg. 237-269 and Pennsylvania University, Philadelphia, U.S.A. UPR-0435T.

[28] J.R. Ellis, S. Kelley and D.V. Nanopoulos, Probing the desert using gauge coupling unification, Phys. Lett. B 260 (1991) 131 [InSPIRE].

[29] U. Amaldi, W. de Boer and H. Furstenau, Comparison of grand unified theories with electroweak and strong coupling constants measured at LEP, Phys. Lett. B 260 (1991) 447 [INSPIRE].

[30] P. Langacker and M.-x. Luo, Implications of precision electroweak experiments for $M_{t}, \rho_{0}$, $\sin ^{2} \theta_{W}$ and grand unification, Phys. Rev. D 44 (1991) 817 [INSPIRE].

[31] C. Giunti, C.W. Kim and U.W. Lee, Running coupling constants and grand unification models, Mod. Phys. Lett. A 6 (1991) 1745 [InSPIRE].

[32] H. Georgi and C. Jarlskog, A New Lepton-Quark Mass Relation in a Unified Theory, Phys. Lett. B 86 (1979) 297 [INSPIRE].

[33] S. Antusch and M. Spinrath, New GUT predictions for quark and lepton mass ratios confronted with phenomenology, Phys. Rev. D 79 (2009) 095004 [arXiv:0902.4644] [INSPIRE].

[34] S. Antusch, S.F. King and M. Spinrath, GUT predictions for quark-lepton Yukawa coupling ratios with messenger masses from non-singlets, Phys. Rev. D 89 (2014) 055027 [arXiv:1311.0877] [INSPIRE].

[35] G. Altarelli and F. Feruglio, Discrete Flavor Symmetries and Models of Neutrino Mixing, Rev. Mod. Phys. 82 (2010) 2701 [arXiv: 1002.0211] [INSPIRE].

[36] H. Ishimori, T. Kobayashi, H. Ohki, Y. Shimizu, H. Okada and M. Tanimoto, Non-Abelian Discrete Symmetries in Particle Physics, Prog. Theor. Phys. Suppl. 183 (2010) 1 [arXiv: 1003.3552] [INSPIRE].

[37] S.F. King and C. Luhn, Neutrino Mass and Mixing with Discrete Symmetry, Rept. Prog. Phys. 76 (2013) 056201 [arXiv: 1301.1340] [INSPIRE].

[38] S.F. King, A. Merle, S. Morisi, Y. Shimizu and M. Tanimoto, Neutrino Mass and Mixing: from Theory to Experiment, New J. Phys. 16 (2014) 045018 [arXiv: 1402.4271] [InSPIRE].

[39] S.F. King, Models of Neutrino Mass, Mixing and CP-violation, J. Phys. G 42 (2015) 123001 [arXiv: 1510.02091] [INSPIRE]. 
[40] S.F. King, Unified Models of Neutrinos, Flavour and CP-violation, Prog. Part. Nucl. Phys. 94 (2017) 217 [arXiv: 1701.04413] [INSPIRE].

[41] F. Björkeroth, F.J. de Anda, I. de Medeiros Varzielas and S.F. King, Towards a complete $A_{4} \times \mathrm{SU}(5) S U S Y$ GUT, JHEP 06 (2015) 141 [arXiv:1503.03306] [INSPIRE].

[42] I.K. Cooper, S.F. King and C. Luhn, A4xSU(5) SUSY GUT of Flavour with Trimaximal Neutrino Mixing, JHEP 06 (2012) 130 [arXiv:1203.1324] [INSPIRE].

[43] S. Antusch, S.F. King and M. Spinrath, Spontaneous CP-violation in $A_{4} \times \mathrm{SU}(5)$ with Constrained Sequential Dominance 2, Phys. Rev. D 87 (2013) 096018 [arXiv:1301.6764] [INSPIRE].

[44] R.A. Laamara, M.A. Loualidi, M. Miskaoui and E.H. Saidi, Hybrid seesaw neutrino model in SUSY SU(5) $\times \mathbb{A}_{4}$, Phys. Rev. D 98 (2018) 015004 [arXiv: 1806.08573] [InSPIRE].

[45] P. Ciafaloni, M. Picariello, E. Torrente-Lujan and A. Urbano, Neutrino masses and tribimaximal mixing in Minimal renormalizable SUSY SU(5) Grand Unified Model with $A_{4}$ Flavor symmetry, Phys. Rev. D 79 (2009) 116010 [arXiv:0901.2236] [InSPIRE].

[46] G. Altarelli, F. Feruglio and C. Hagedorn, A SUSY SU(5) Grand Unified Model of Tri-Bimaximal Mixing from $A_{4}$, JHEP 03 (2008) 052 [arXiv:0802.0090] [INSPIRE].

[47] S. Antusch, S.F. King and M. Spinrath, Measurable Neutrino Mass Scale in $A_{4} \times \mathrm{SU}(5)$, Phys. Rev. D 83 (2011) 013005 [arXiv: 1005.0708] [INSPIRE].

[48] I.K. Cooper, S.F. King and C. Luhn, SUSY SU(5) with singlet plus adjoint matter and A4 family symmetry, Phys. Lett. B 690 (2010) 396 [arXiv:1004.3243] [INSPIRE].

[49] R. Ahl Laamara, M.A. Loualidi, M. Miskaoui and E.H. Saidi, Fermion masses and mixing in $\mathrm{SU}(5) \times D_{4} \times \mathrm{U}(1)$ model, Nucl. Phys. B 916 (2017) 430 [inSPIRE].

[50] W. Grimus and L. Lavoura, A discrete symmetry group for maximal atmospheric neutrino mixing, Phys. Lett. B 572 (2003) 189 [hep-ph/0305046] [INSPIRE].

[51] T. Fukuyama and H. Nishiura, Mass matrix of Majorana neutrinos, hep-ph/9702253 [INSPIRE].

[52] E. Ma and M. Raidal, Neutrino mass, muon anomalous magnetic moment, and lepton flavor nonconservation, Phys. Rev. Lett. 87 (2001) 011802 [Erratum ibid. 87 (2001) 159901] [hep-ph/0102255] [INSPIRE].

[53] C.S. Lam, A 2-3 symmetry in neutrino oscillations, Phys. Lett. B 507 (2001) 214 [hep-ph/0104116] [INSPIRE].

[54] K.R.S. Balaji, W. Grimus and T. Schwetz, The solar LMA neutrino oscillation solution in the Zee model, Phys. Lett. B 508 (2001) 301 [hep-ph/0104035] [InSPIRE].

[55] P.F. Harrison and W.G. Scott, $\mu-\tau$ reflection symmetry in lepton mixing and neutrino oscillations, Phys. Lett. B 547 (2002) 219 [hep-ph/0210197] [INSPIRE].

[56] D. Bödeker and W. Buchmüller, Baryogenesis from the weak scale to the grand unification scale, Rev. Mod. Phys. 93 (2021) 035004 [arXiv:2009.07294] [INSPIRE].

[57] C.S. Fong, M.H. Rahat and S. Saad, Low-scale Resonant Leptogenesis in SU(5) GUT with $\mathcal{T}_{13}$ Family Symmetry, arXiv:2103.14691 [INSPIRE].

[58] M.H. Rahat, Leptogenesis from the Asymmetric Texture, Phys. Rev. D 103 (2021) 035011 [arXiv: 2008. 04204] [INSPIRE]. 
[59] M.K. Parida and R. Samantaray, SU(5) unification of two triplet seesaw and leptogenesis with dark matter and vacuum stability, Nucl. Phys. B 970 (2021) 115484

[arXiv: 2102.01605] [INSPIRE].

[60] J. Gehrlein, S.T. Petcov, M. Spinrath and X. Zhang, Leptogenesis in an SU(5) $\times A_{5}$ Golden Ratio Flavour Model: Addendum, arXiv:1508.07930 [Addendum ibid. 899 (2015) 617] [INSPIRE].

[61] A. Meroni, E. Molinaro and S.T. Petcov, Revisiting Leptogenesis in a SUSY SU(5) $\times T^{\prime}$ Model of Flavour, Phys. Lett. B 710 (2012) 435 [arXiv: 1203.4435] [INSPIRE].

[62] S. Antusch and V. Maurer, Running quark and lepton parameters at various scales, JHEP 11 (2013) 115 [arXiv: 1306.6879] [INSPIRE].

[63] C.S. Lam, Mass Independent Textures and Symmetry, Phys. Rev. D 74 (2006) 113004 [hep-ph/0611017] [INSPIRE].

[64] J.D. Bjorken, P.F. Harrison and W.G. Scott, Simplified unitarity triangles for the lepton sector, Phys. Rev. D 74 (2006) 073012 [hep-ph/0511201] [INSPIRE].

[65] Z.-z. Xing and S. Zhou, Tri-bimaximal Neutrino Mixing and Flavor-dependent Resonant Leptogenesis, Phys. Lett. B 653 (2007) 278 [hep-ph/0607302] [INSPIRE].

[66] X.-G. He and A. Zee, Minimal modification to the tri-bimaximal neutrino mixing, Phys. Lett. B 645 (2007) 427 [hep-ph/0607163] [INSPIRE].

[67] W. Grimus and L. Lavoura, A model for trimaximal lepton mixing, JHEP 09 (2008) 106 [arXiv: 0809.0226] [INSPIRE].

[68] W. Grimus and L. Lavoura, A Three-parameter neutrino mass matrix with maximal CP-violation, Phys. Lett. B 671 (2009) 456 [arXiv:0810.4516] [INSPIRE].

[69] C.H. Albright and W. Rodejohann, Comparing Trimaximal Mixing and Its Variants with Deviations from Tri-bimaximal Mixing, Eur. Phys. J. C 62 (2009) 599 [arXiv:0812.0436] [INSPIRE].

[70] C.H. Albright, A. Dueck and W. Rodejohann, Possible Alternatives to Tri-bimaximal Mixing, Eur. Phys. J. C 70 (2010) 1099 [arXiv: 1004.2798] [InSPIRE].

[71] E.E. Jenkins and A.V. Manohar, Tribimaximal Mixing, Leptogenesis and $\theta_{13}$, Phys. Lett. B 668 (2008) 210 [arXiv:0807.4176] [INSPIRE].

[72] B. Karmakar and A. Sil, Nonzero $\theta_{13}$ and leptogenesis in a type-I seesaw model with $A_{4}$ symmetry, Phys. Rev. D 91 (2015) 013004 [arXiv:1407.5826] [INSPIRE].

[73] Planck collaboration, Planck 2018 results. VI. Cosmological parameters, Astron. Astrophys. 641 (2020) A6 [Erratum ibid. 652 (2021) C4] [arXiv:1807.06209] [INSPIRE].

[74] S. Vagnozzi et al., Unveiling $\nu$ secrets with cosmological data: neutrino masses and mass hierarchy, Phys. Rev. D 96 (2017) 123503 [arXiv:1701.08172] [INSPIRE].

[75] P.F. Harrison, D.H. Perkins and W.G. Scott, Tri-bimaximal mixing and the neutrino oscillation data, Phys. Lett. B 530 (2002) 167 [hep-ph/0202074] [InSPIRE].

[76] L.J. Hall, R. Rattazzi and U. Sarid, The top quark mass in supersymmetric $\mathrm{SO}(10)$ unification, Phys. Rev. D 50 (1994) 7048 [hep-ph/9306309] [INSPIRE].

[77] M. Carena, M. Olechowski, S. Pokorski and C.E.M. Wagner, Electroweak symmetry breaking and bottom-top Yukawa unification, Nucl. Phys. B 426 (1994) 269 [hep-ph/9402253] [INSPIRE]. 
[78] R. Hempfling, Yukawa coupling unification with supersymmetric threshold corrections, Phys. Rev. D 49 (1994) 6168.

[79] T. Blazek, S. Raby and S. Pokorski, Finite supersymmetric threshold corrections to CKM matrix elements in the large $\tan \beta$ regime, Phys. Rev. D 52 (1995) 4151 [hep-ph/9504364] [INSPIRE].

[80] H. Fusaoka and Y. Koide, Updated estimate of running quark masses, Phys. Rev. D $5 \mathbf{7}$ (1998) 3986 [hep-ph/9712201] [INSPIRE].

[81] Z.-z. Xing, H. Zhang and S. Zhou, Updated Values of Running Quark and Lepton Masses, Phys. Rev. D 77 (2008) 113016 [arXiv:0712.1419] [InSPIRE].

[82] S. Antusch, J. Kersten, M. Lindner, M. Ratz and M.A. Schmidt, Running neutrino mass parameters in see-saw scenarios, JHEP 03 (2005) 024 [hep-ph/0501272] [INSPIRE].

[83] Super-Kamiokande collaboration, Search for Nucleon Decay in Super-Kamiokande, Nucl. Part. Phys. Proc. 516 (2016) 273.

[84] G.R. Farrar and P. Fayet, Phenomenology of the Production, Decay, and Detection of New Hadronic States Associated with Supersymmetry, Phys. Lett. B 76 (1978) 575 [inSPIRE].

[85] N. Sakai and T. Yanagida, Proton Decay in a Class of Supersymmetric Grand Unified Models, Nucl. Phys. B 197 (1982) 533 [InSPIRE].

[86] P. Nath and R.L. Arnowitt, Limits on Photino and Squark Masses From Proton Lifetime in Supergravity and Superstring Models, Phys. Rev. D 38 (1988) 1479 [inSPIRE].

[87] D. Emmanuel-Costa and S. Wiesenfeldt, Proton decay in a consistent supersymmetric SU(5) GUT model, Nucl. Phys. B 661 (2003) 62 [hep-ph/0302272] [INSPIRE].

[88] R. Harnik, D.T. Larson, H. Murayama and M. Thormeier, Probing the Planck scale with proton decay, Nucl. Phys. B 706 (2005) 372 [hep-ph/0404260] [INSPIRE].

[89] P. Nath, A.H. Chamseddine and R.L. Arnowitt, Nucleon Decay in Supergravity Unified Theories, Phys. Rev. D 32 (1985) 2348 [InSPIRE].

[90] A. Masiero, D.V. Nanopoulos, K. Tamvakis and T. Yanagida, Naturally Massless Higgs Doublets in Supersymmetric SU(5), Phys. Lett. B 115 (1982) 380 [InSPIRE].

[91] B. Grinstein, A Supersymmetric SU(5) Gauge Theory with No Gauge Hierarchy Problem, Nucl. Phys. B 206 (1982) 387 [inSPIRE].

[92] J. Hisano, T. Moroi, K. Tobe and T. Yanagida, Suppression of proton decay in the missing partner model for supersymmetric SU(5) GUT, Phys. Lett. B 342 (1995) 138 [hep-ph/9406417] [INSPIRE].

[93] S. Antusch, I. de Medeiros Varzielas, V. Maurer, C. Sluka and M. Spinrath, Towards predictive flavour models in SUSY SU(5) GUTs with doublet-triplet splitting, JHEP 09 (2014) 141 [arXiv:1405.6962] [INSPIRE].

[94] Particle Data Group collaboration, Review of Particle Physics, PTEP 2020 (2020) 083C01 [INSPIRE].

[95] KATRIN collaboration, Improved Upper Limit on the Neutrino Mass from a Direct Kinematic Method by KATRIN, Phys. Rev. Lett. 123 (2019) 221802 [arXiv:1909.06048] [INSPIRE].

[96] KATRIN collaboration, KATRIN design report 2004, https://www.katrin.kit.edu/publikationen/DesignReport2004-12Jan2005.pdf. 
[97] CORE collaboration, Exploring cosmic origins with CORE: Cosmological parameters, JCAP 04 (2018) 017 [arXiv: 1612.00021] [INSPIRE].

[98] B. Alpert et al., HOLMES - The Electron Capture Decay of ${ }^{163}$ Ho to Measure the Electron Neutrino Mass with sub-eV sensitivity, Eur. Phys. J. C 75 (2015) 112 [arXiv:1412.5060] [INSPIRE].

[99] Project 8 collaboration, Determining the neutrino mass with cyclotron radiation emission spectroscopy - Project 8, J. Phys. G 44 (2017) 054004 [arXiv: 1703. 02037] [INSPIRE].

[100] KamLAND-Zen collaboration, Search for Majorana Neutrinos near the Inverted Mass Hierarchy Region with KamLAND-Zen, Phys. Rev. Lett. 117 (2016) 082503 [Addendum ibid. 117 (2016) 109903] [arXiv: 1605. 02889] [INSPIRE].

[101] CUORE collaboration, First Results from CUORE: A Search for Lepton Number Violation via $0 \nu \beta \beta$ Decay of ${ }^{130}$ Te, Phys. Rev. Lett. 120 (2018) 132501 [arXiv:1710.07988] [INSPIRE].

[102] GERDA collaboration, Results on Neutrinoless Double- $\beta$ Decay of ${ }^{76}$ Ge from Phase I of the GERDA Experiment, Phys. Rev. Lett. 111 (2013) 122503 [arXiv:1307.4720] [InSPIRE].

[103] EXO-200 collaboration, Search for Neutrinoless Double- $\beta$ Decay with the Complete EXO-200 Dataset, Phys. Rev. Lett. 123 (2019) 161802 [arXiv:1906. 02723] [INSPIRE].

[104] M. Agostini et al., Background-free search for neutrinoless double- $\beta$ decay of ${ }^{76}$ Ge with GERDA, Nature 544 (2017) 47 [arXiv:1703.00570] [INSPIRE].

[105] CUPID collaboration, CUPID: CUORE (Cryogenic Underground Observatory for Rare Events) Upgrade with Particle IDentification, arXiv:1504.03599 [INSPIRE].

[106] NEXO collaboration, Sensitivity and Discovery Potential of nEXO to Neutrinoless Double Beta Decay, Phys. Rev. C 97 (2018) 065503 [arXiv:1710.05075] [InSPIRE].

[107] SNO+ collaboration, Current Status and Future Prospects of the SNO+ Experiment, Adv. High Energy Phys. 2016 (2016) 6194250 [arXiv: 1508.05759] [INSPIRE].

[108] S. Davidson, E. Nardi and Y. Nir, Leptogenesis, Phys. Rept. 466 (2008) 105 [arXiv:0802.2962] [INSPIRE].

[109] C.S. Fong, E. Nardi and A. Riotto, Leptogenesis in the Universe, Adv. High Energy Phys. 2012 (2012) 158303 [arXiv:1301.3062] [INSPIRE].

[110] G.F. Giudice, A. Notari, M. Raidal, A. Riotto and A. Strumia, Towards a complete theory of thermal leptogenesis in the SM and MSSM, Nucl. Phys. B 685 (2004) 89 [hep-ph/0310123] [INSPIRE].

[111] C.S. Fong, M.C. Gonzalez-Garcia, E. Nardi and J. Racker, Supersymmetric Leptogenesis, JCAP 12 (2010) 013 [arXiv: 1009.0003] [INSPIRE].

[112] Y. Grossman, T. Kashti, Y. Nir and E. Roulet, Leptogenesis from supersymmetry breaking, Phys. Rev. Lett.textbf91 (2003) 251801 [arXiv:0307081].

[113] G. D'Ambrosio, G.F. Giudice and M. Raidal, Soft leptogenesis, Phys. Lett. B 575 (2003) 75 [hep-ph/0308031] [INSPIRE].

[114] J.A. Harvey and M.S. Turner, Cosmological baryon and lepton number in the presence of electroweak fermion number violation, Phys. Rev. D 42 (1990) 3344 [INSPIRE].

[115] G. Altarelli and F. Feruglio, Tri-bimaximal neutrino mixing, $A_{4}$ and the modular symmetry, Nucl. Phys. B 741 (2006) 215 [hep-ph/0512103] [INSPIRE]. 